\title{
Standard Model Higgs results from ATLAS and CMS experiments
}

\section{Jana Faltova}

\section{on behalf of ATLAS and CMS Collaborations}
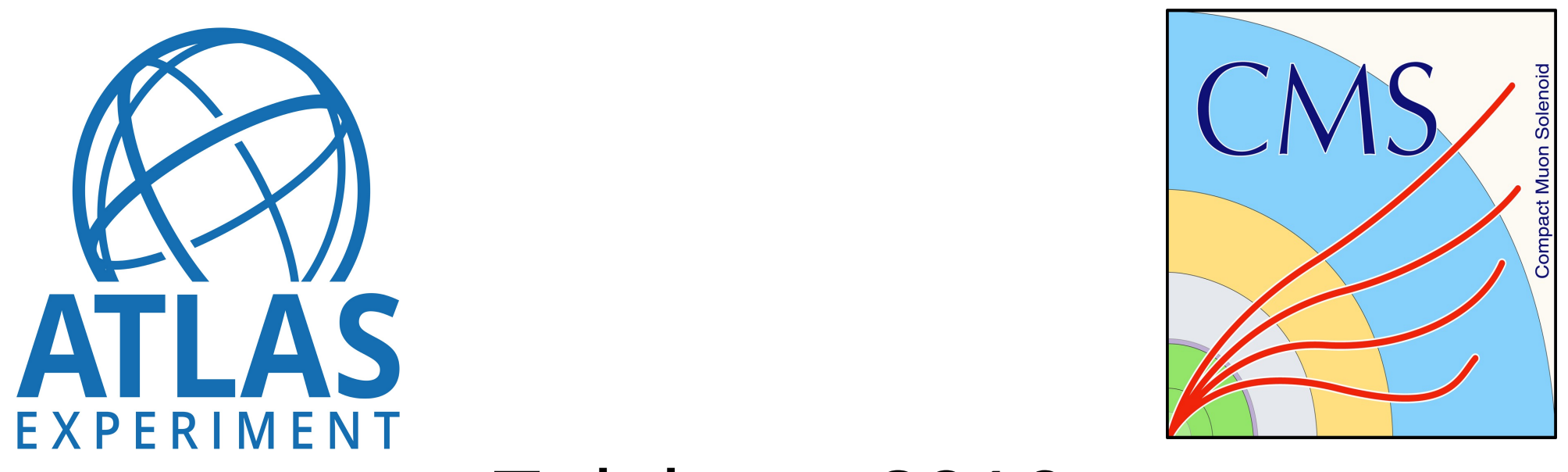

Epiphany 2016

7 - 9 January 2016, Krakow 


\section{Outline}

- LHC collider

- ATLAS, CMS detectors

- Run1 and Run2 conditions

- Higgs boson

- Run1 results

- First Run2 measurements

- Conclusions 


\section{Large Hadron Collider (LHC)}

LHC at CERN

- $27 \mathrm{~km}$ long ring

- proton-proton and heavy ion collisions

Running conditions

- Run1 (2010 - 2012)

- $\sqrt{ } \mathrm{s}=7,8 \mathrm{TeV}$

- 50 ns bunch spacing

- Run2 (since 2015)

- $\sqrt{s}=13 \mathrm{TeV}$

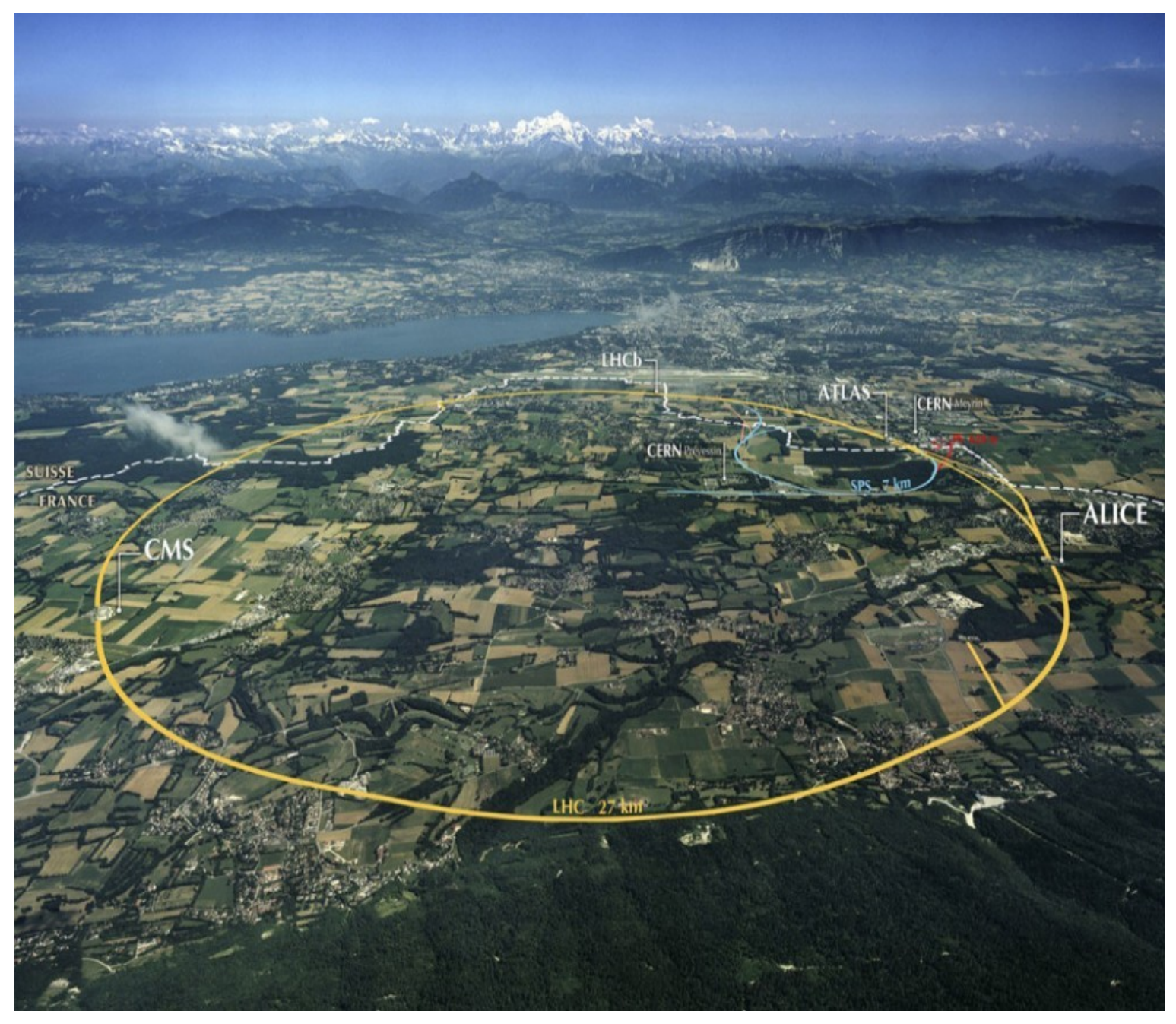

- 50 and 25 ns bunch spacing 


\section{ATLAS and CMS detectors}

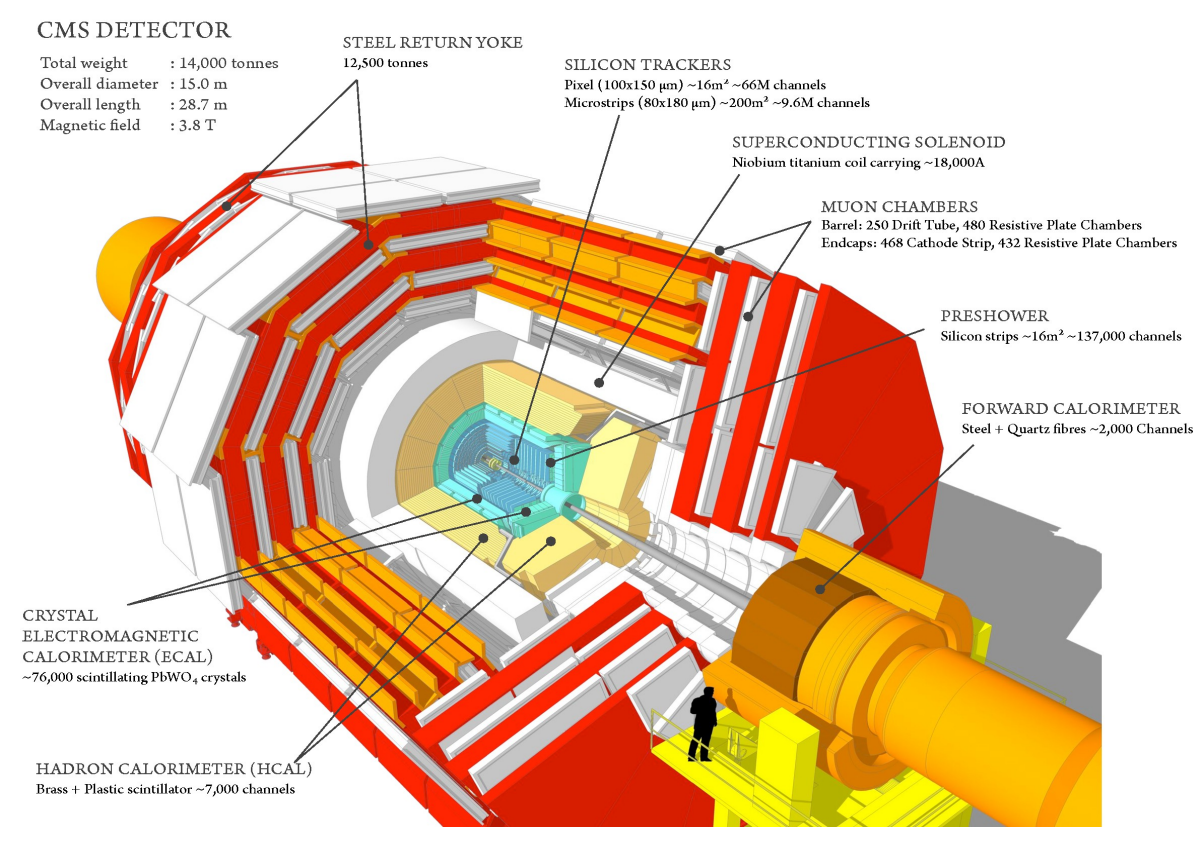

CMS in Run2

- Cold operation of Tracker

- Luminosity detectors

- 4th muon station

- New beam pipe

ATLAS in Run2

- 4th inner most layer of pixels $(3.3 \mathrm{~cm})$

- Complete muon coverage

- Luminosity detectors

- Trigger 


\section{Luminosity in Run1 and Run2}
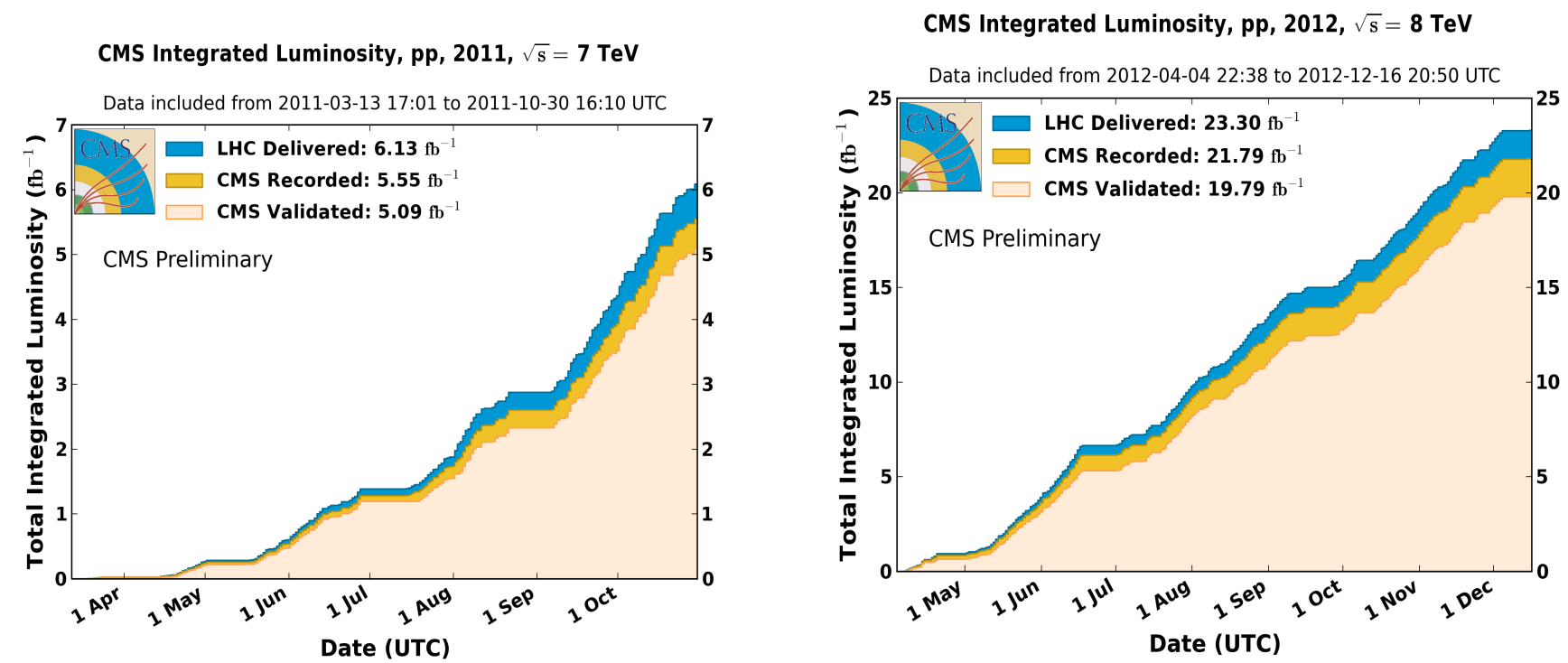

CMS Integrated Luminosity, pp, 2015, $\sqrt{\mathrm{s}}=13 \mathrm{TeV}$

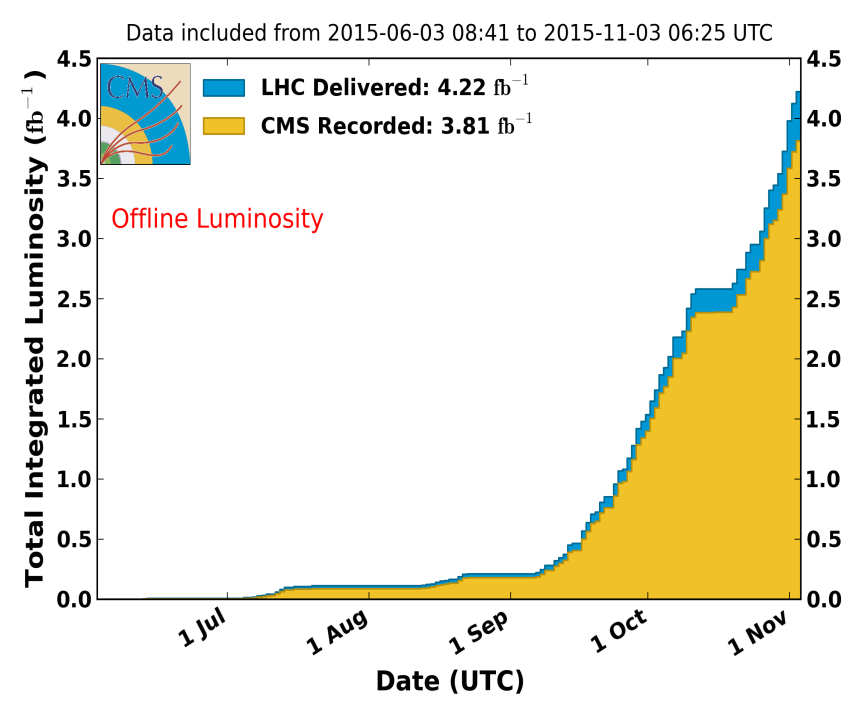




\section{Luminosity in Run1 and Run2}

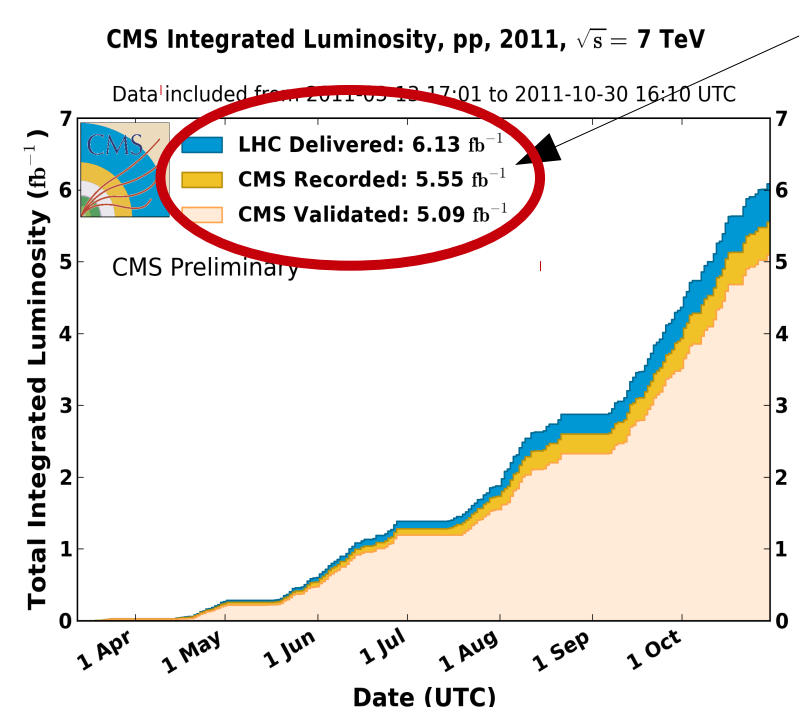

CMS Integrated Luminosity, pp, 2015, $\sqrt{\mathrm{s}}=13 \mathrm{TeV}$

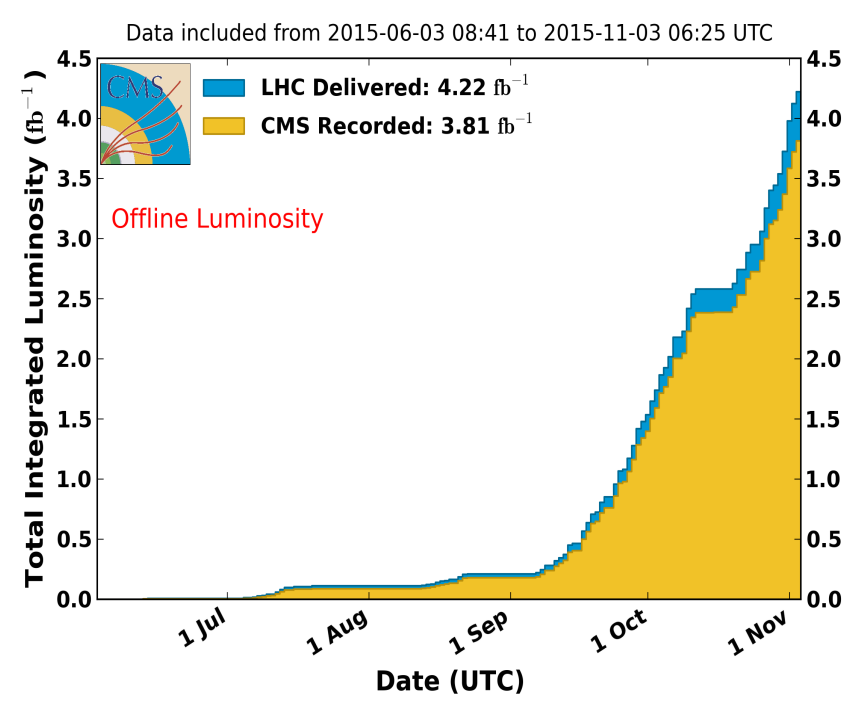

CMS Integrated Luminosity, pp, 2012, $\sqrt{\mathrm{s}}=\mathbf{8} \mathrm{TeV}$

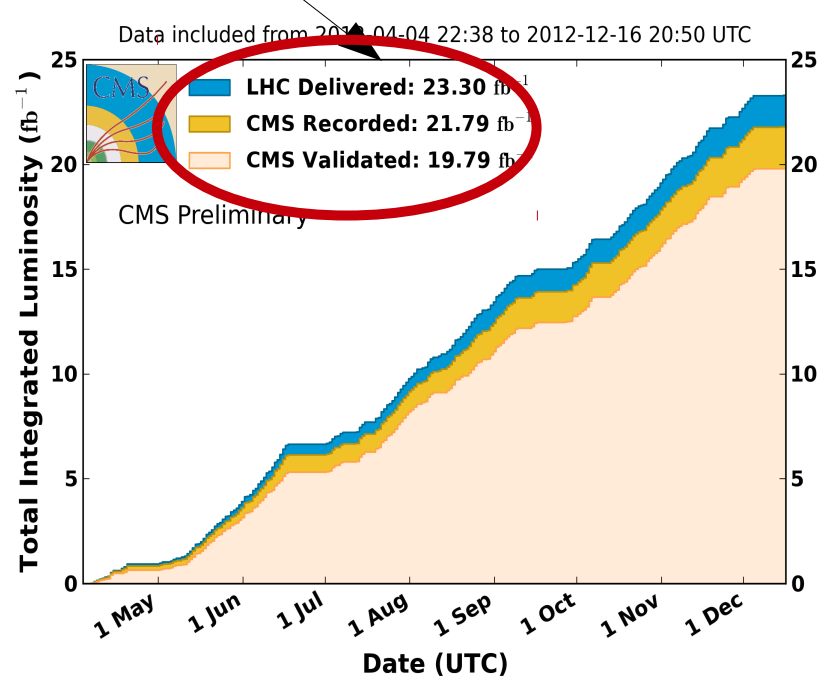

Good for physics analysis in Run1

- ATLAS: $4.6 \mathrm{fb}^{-1}+20.3 \mathrm{fb}^{-1}$

- CMS: $5.1 \mathrm{fb}^{-1}+19.8 \mathrm{fb}^{-1}$ 


\section{Luminosity in Run1 and Run2}
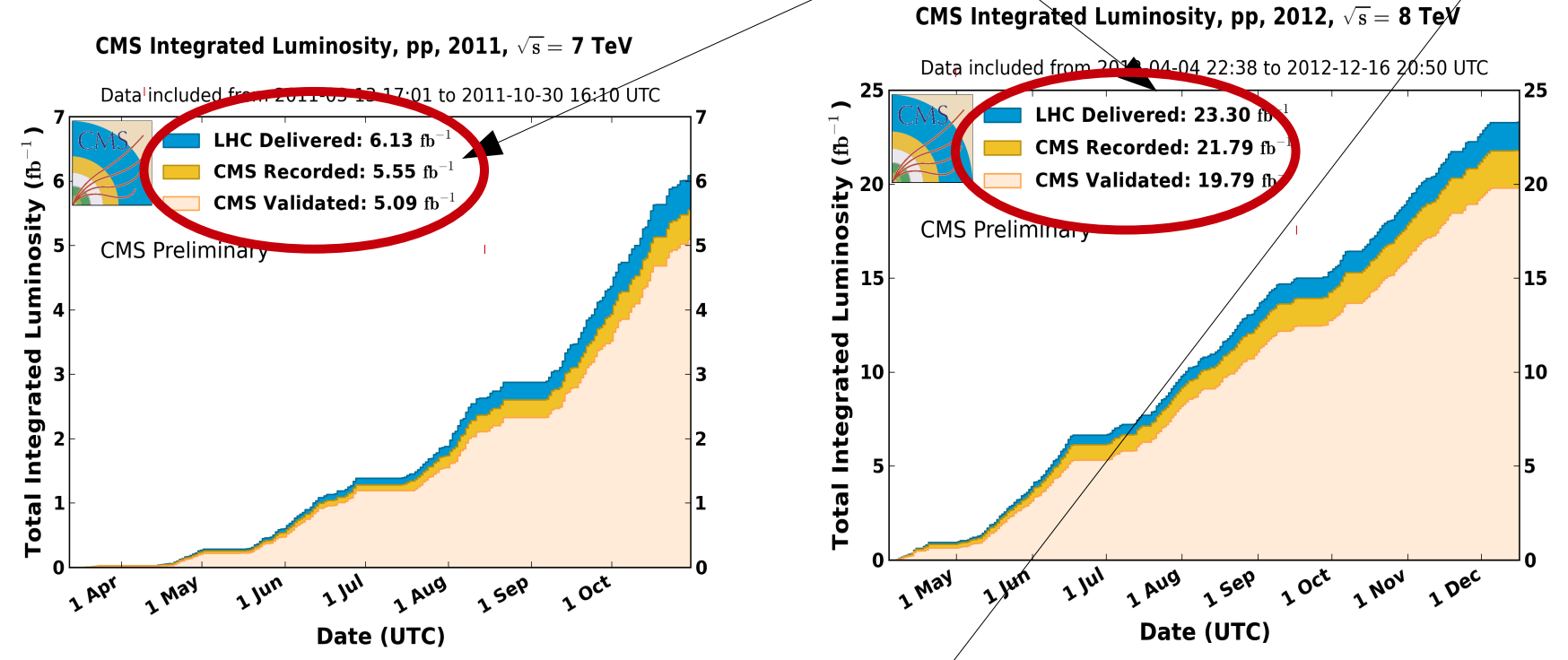

CMS Integrated Luminosity, pp, 2015, $\sqrt{\mathrm{s}}=13 \mathrm{TeV}$

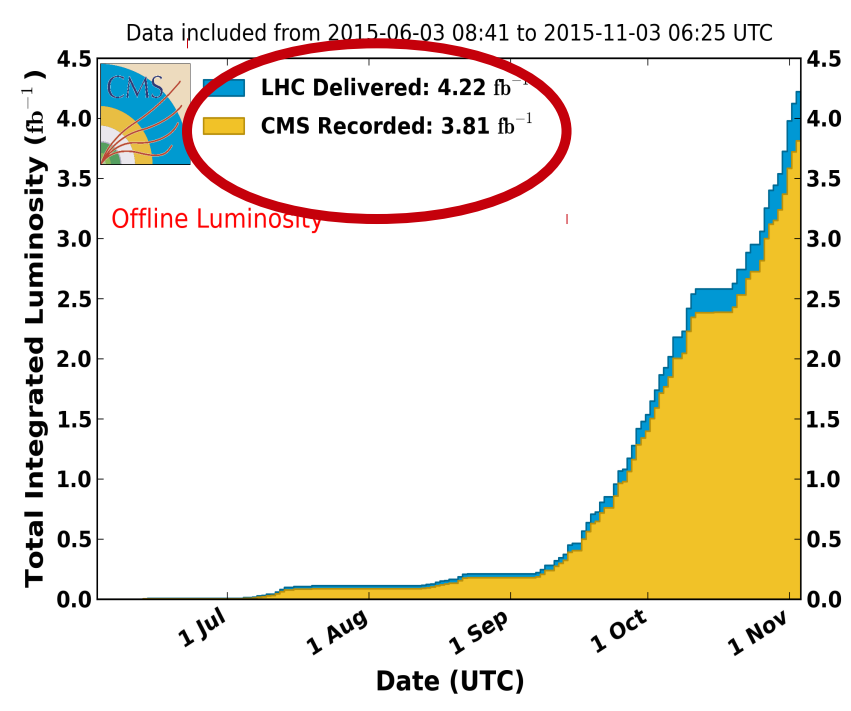

Good for physics analysis in Run1

- ATLAS: $4.6 \mathrm{fb}^{-1}+20.3 \mathrm{fb}^{-1}$

- CMS: $5.1 \mathrm{fb}^{-1}+19.8 \mathrm{fb}^{-1}$

Good for physics analysis in Run2

- ATLAS: $3.2 \mathrm{fb}^{-1}$

- CMS: $2.6 \mathrm{fb}^{-1}$ (magnet cryogenic supply issues) 


\section{Pileup in Run1 and Run2}
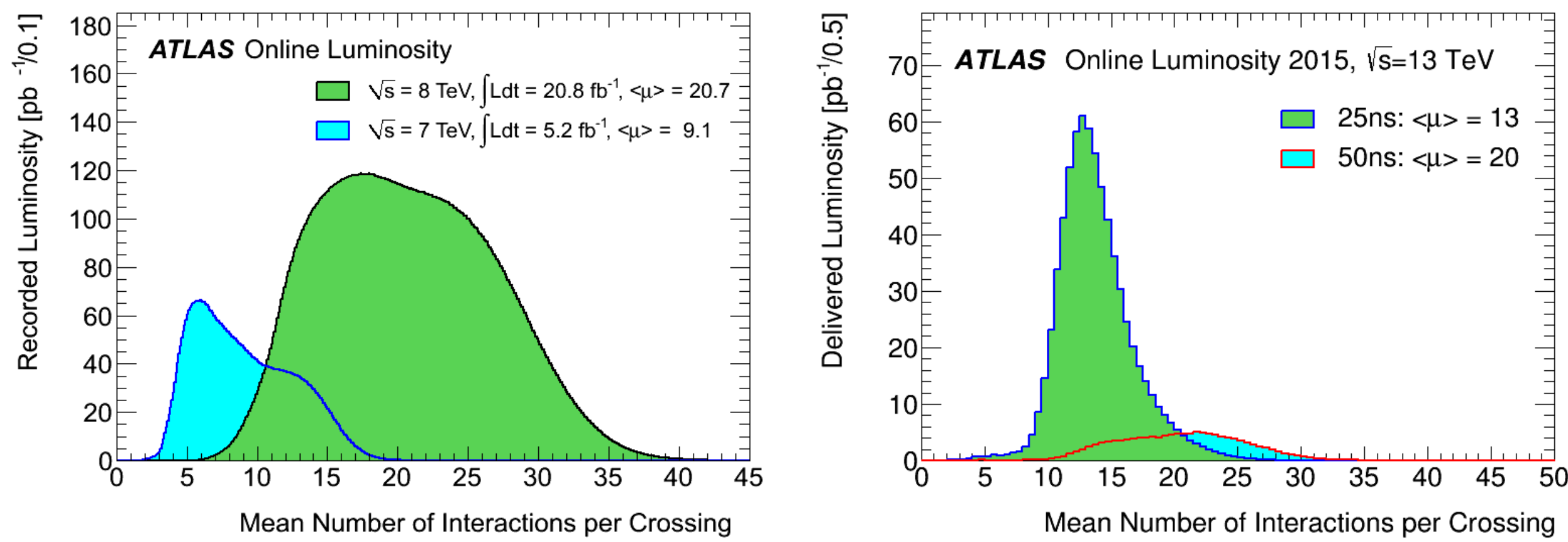


\section{Pileup in Run1 and Run2}
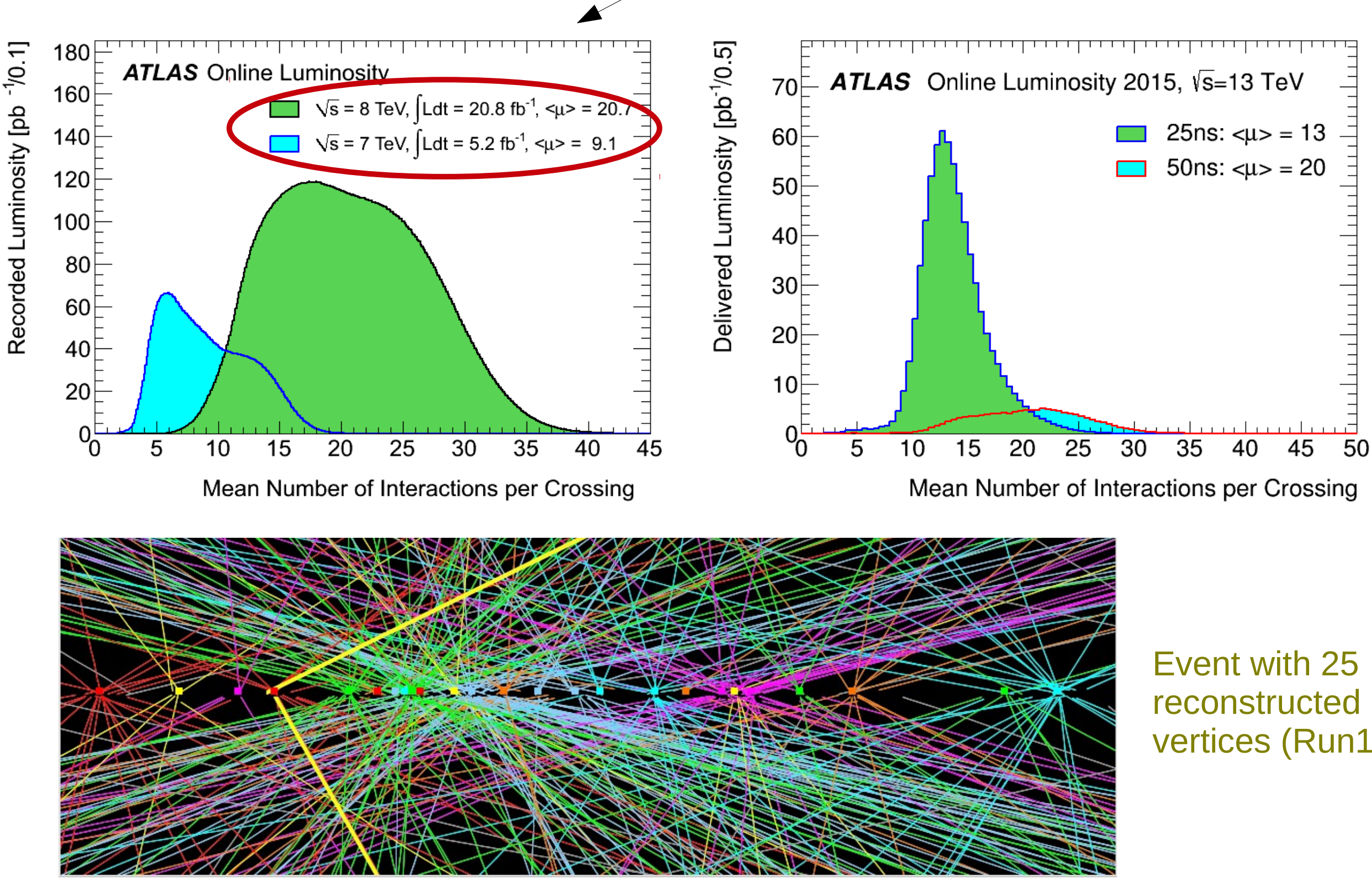

Event with 25 reconstructed

vertices (Run1) 


\section{Pileup in Run1 and Run2}

$\triangle$

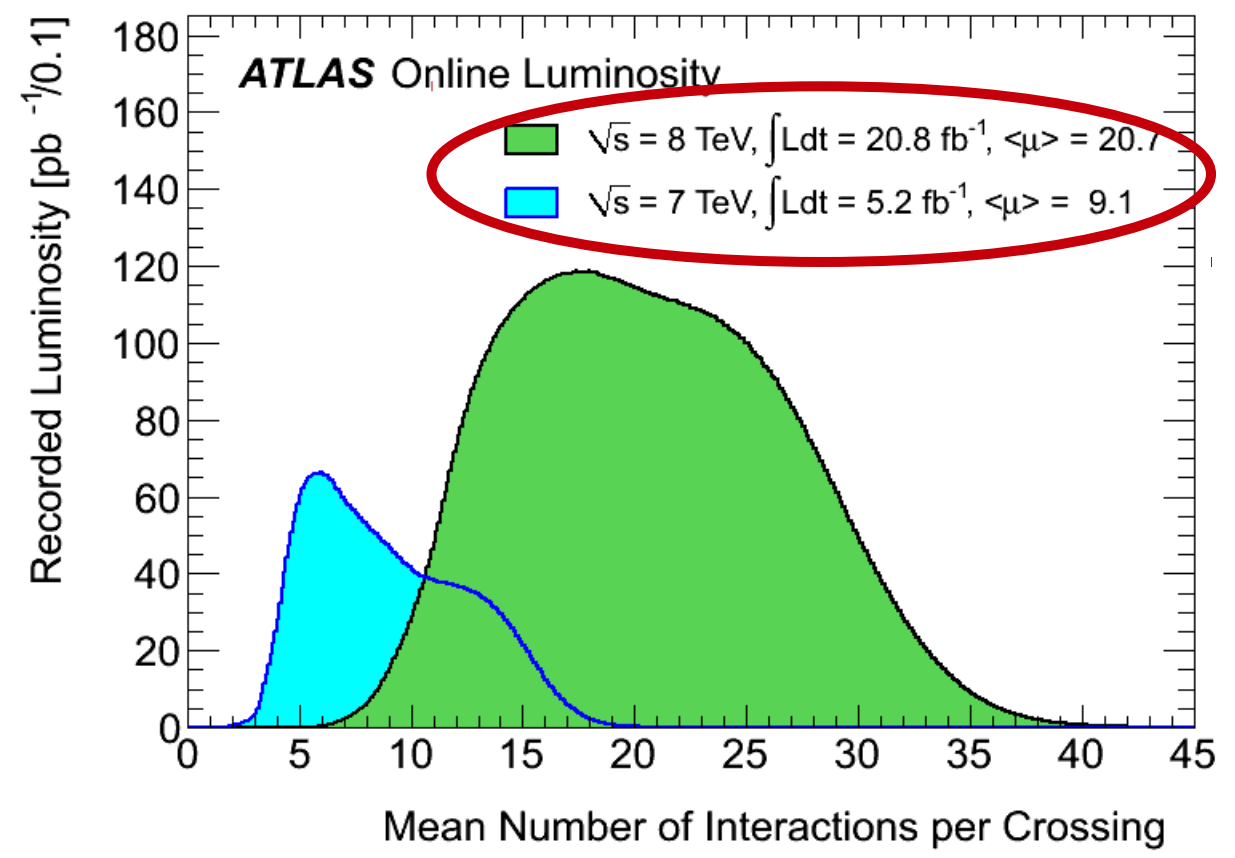

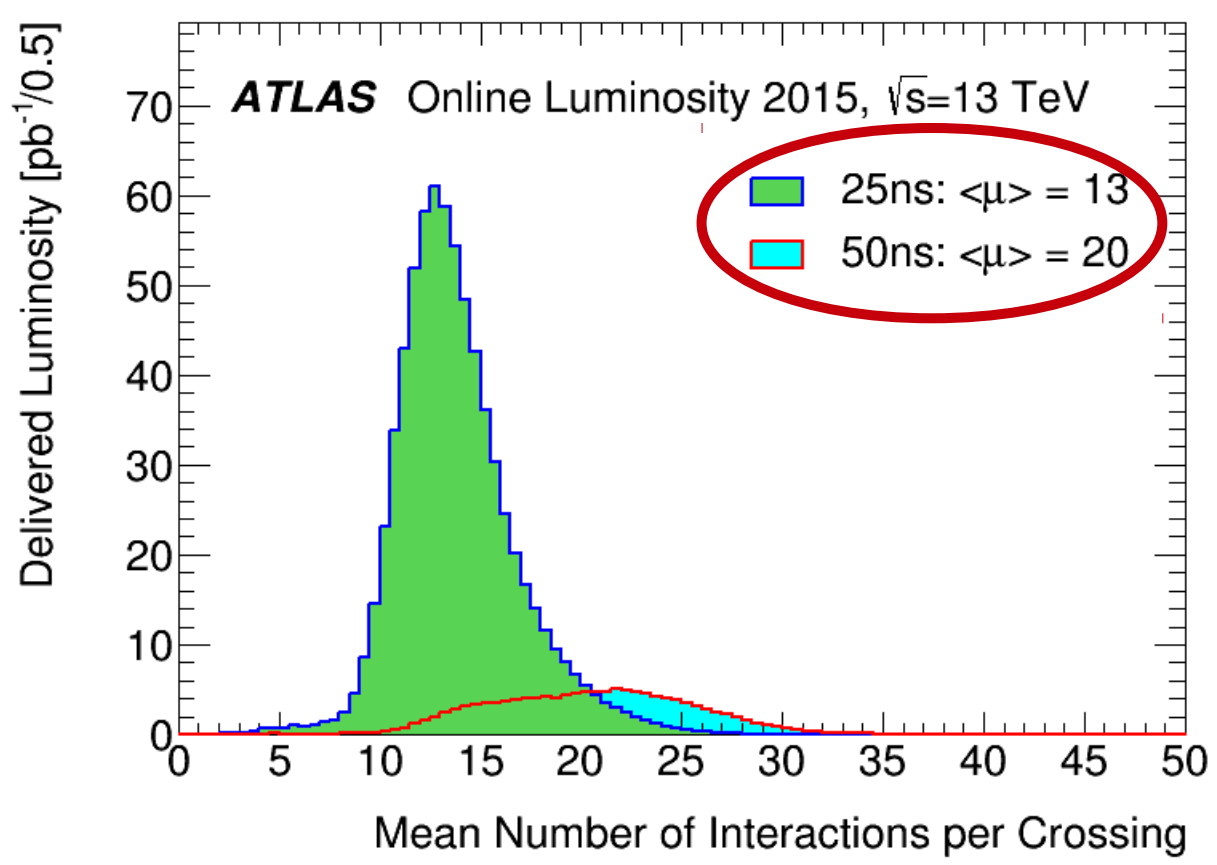

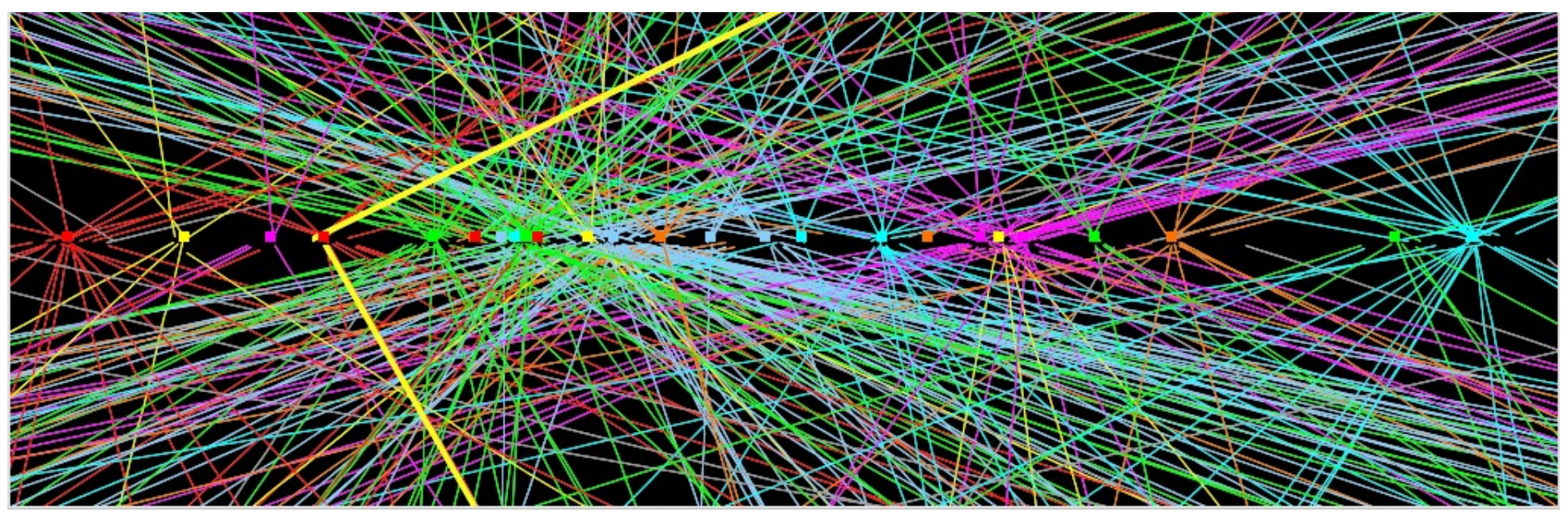

7-9th January
Event with 25 reconstructed vertices (Run1) 


\section{Standard Model (SM) and Higgs boson}

Brout-Englert-Higgs mechanism

- Masses of particles via spontaneous symmetry breaking

- Cancellation of tree level divergences

- Higgs boson particle
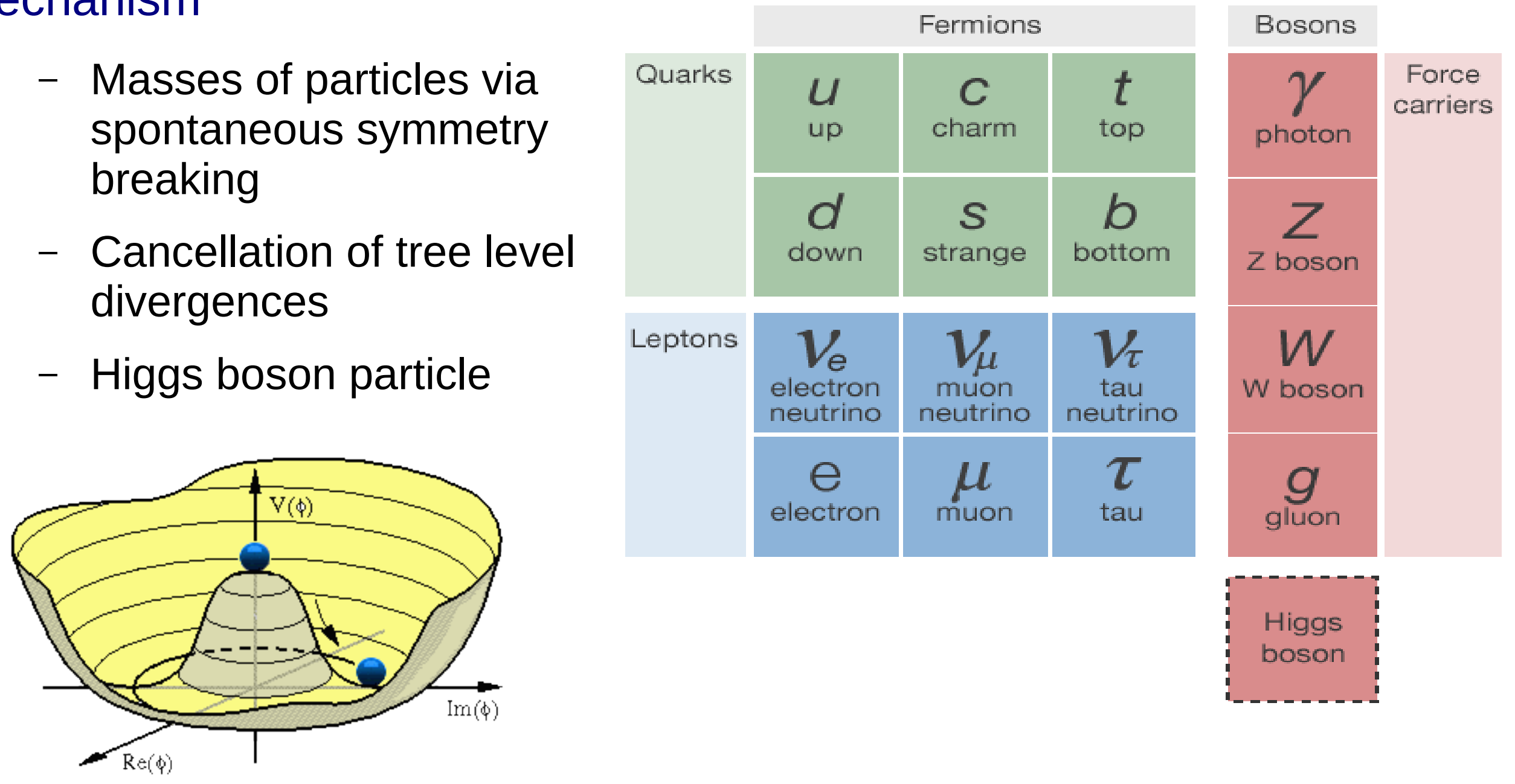


\section{Higgs production at LHC}

\section{Production modes}

- $g g$ fusion

- vector boson fusion

- associated production with $\mathrm{W} / \mathrm{Z}$ boson, bb, tt

\begin{tabular}{|c|c|c|c|}
\hline \multirow{2}{*}{$\begin{array}{c}\text { Production } \\
\text { process }\end{array}$} & \multicolumn{2}{|c|}{ Cross section $[\mathrm{pb}]$} & \multirow{2}{*}{$\begin{array}{c}\text { Order of } \\
\text { calculation }\end{array}$} \\
\hline & $\sqrt{s}=7 \mathrm{TeV}$ & $\sqrt{s}=8 \mathrm{TeV}$ & \\
\hline$g g \mathrm{~F}$ & $15.0 \pm 1.6$ & $19.2 \pm 2.0$ & $\mathrm{NNLO}(\mathrm{QCD})+\mathrm{NLO}(\mathrm{EW})$ \\
\hline$V B F$ & $1.22 \pm 0.03$ & $1.58 \pm 0.04$ & $\mathrm{NLO}(\mathrm{QCD}+\mathrm{EW})+\sim \mathrm{NNLO}(\mathrm{Q}$ \\
\hline$W H$ & $0.577 \pm 0.016$ & $0.703 \pm 0.018$ & NNLO(QUD)+NLO(EN) \\
\hline$Z H$ & $0.334 \pm 0.013$ & $0.414 \pm 0.016$ & $\mathrm{NNLO}(\mathrm{QCD})+\mathrm{NLO}(\mathrm{EW})$ \\
\hline$[g g Z H]$ & $0.023 \pm 0.007$ & $0.032 \pm 0.010$ & $\mathrm{NLO}(\mathrm{QCD})$ \\
\hline$b b H$ & $0.156 \pm 0.021$ & $0.203 \pm 0.028$ & $5 \mathrm{FS} N \mathrm{NLO}(\mathrm{QCD})+4 \mathrm{FS} \mathrm{NLO}(\mathrm{QCD})$ \\
\hline$t t H$ & $0.086 \pm 0.009$ & $0.129 \pm 0.014$ & $\mathrm{NLO}(\mathrm{QCD})$ \\
\hline$t H$ & $0.012 \pm 0.001$ & $0.018 \pm 0.001$ & $\mathrm{NLO}(\mathrm{QCD})$ \\
\hline Total & $17.4 \pm 1.6$ & $22.3 \pm 2.0$ & \\
\hline
\end{tabular}

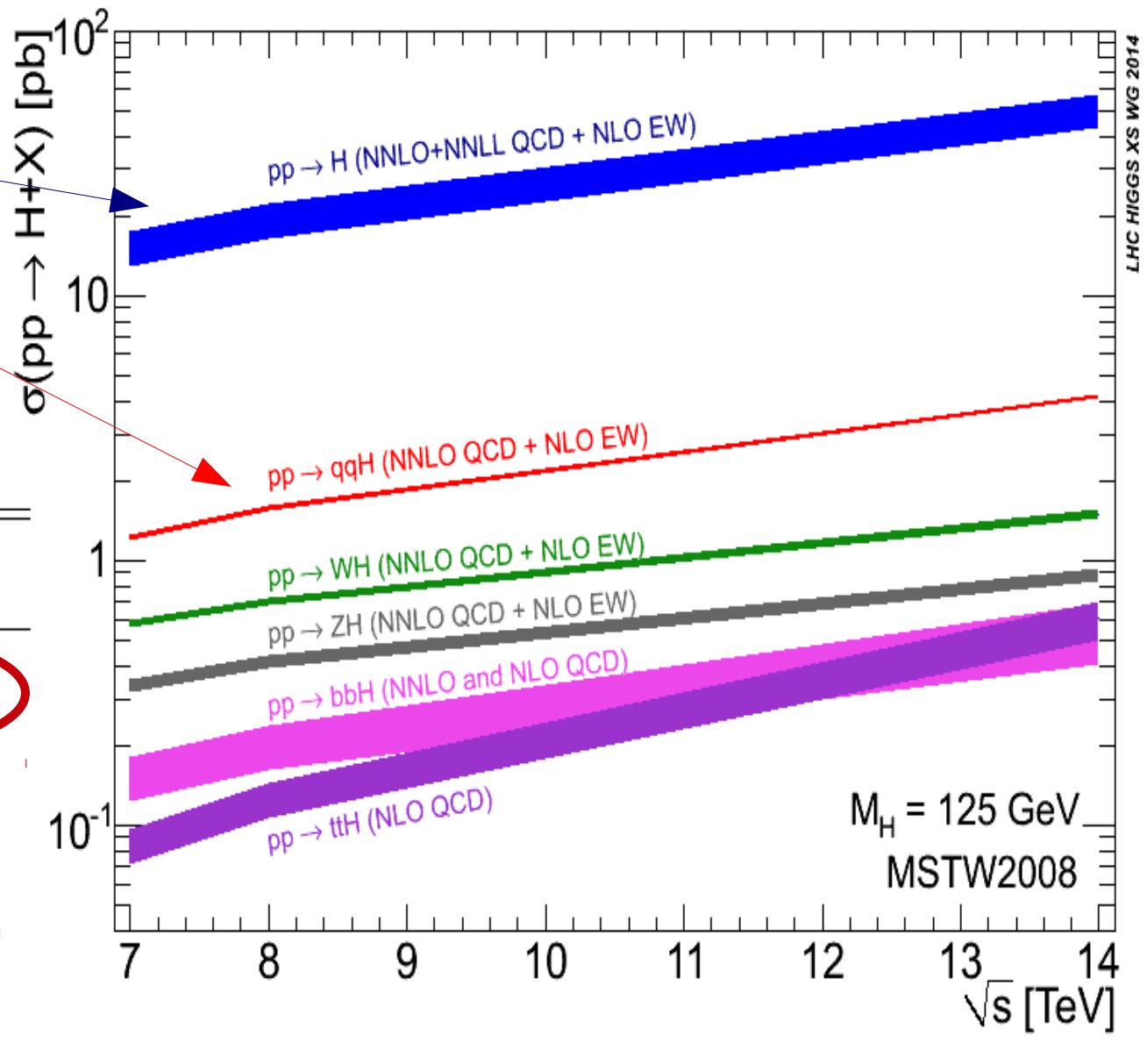




\section{Higgs production at LHC}

Production modes

- gg fusion

- vector boson fusion

- associated production with W/Z boson, bb, tt

Increase by a factor of 2.3 from 8 to $13 \mathrm{TeV}$ in gg fusion

- Even larger increase for $\mathrm{ttH}$ production

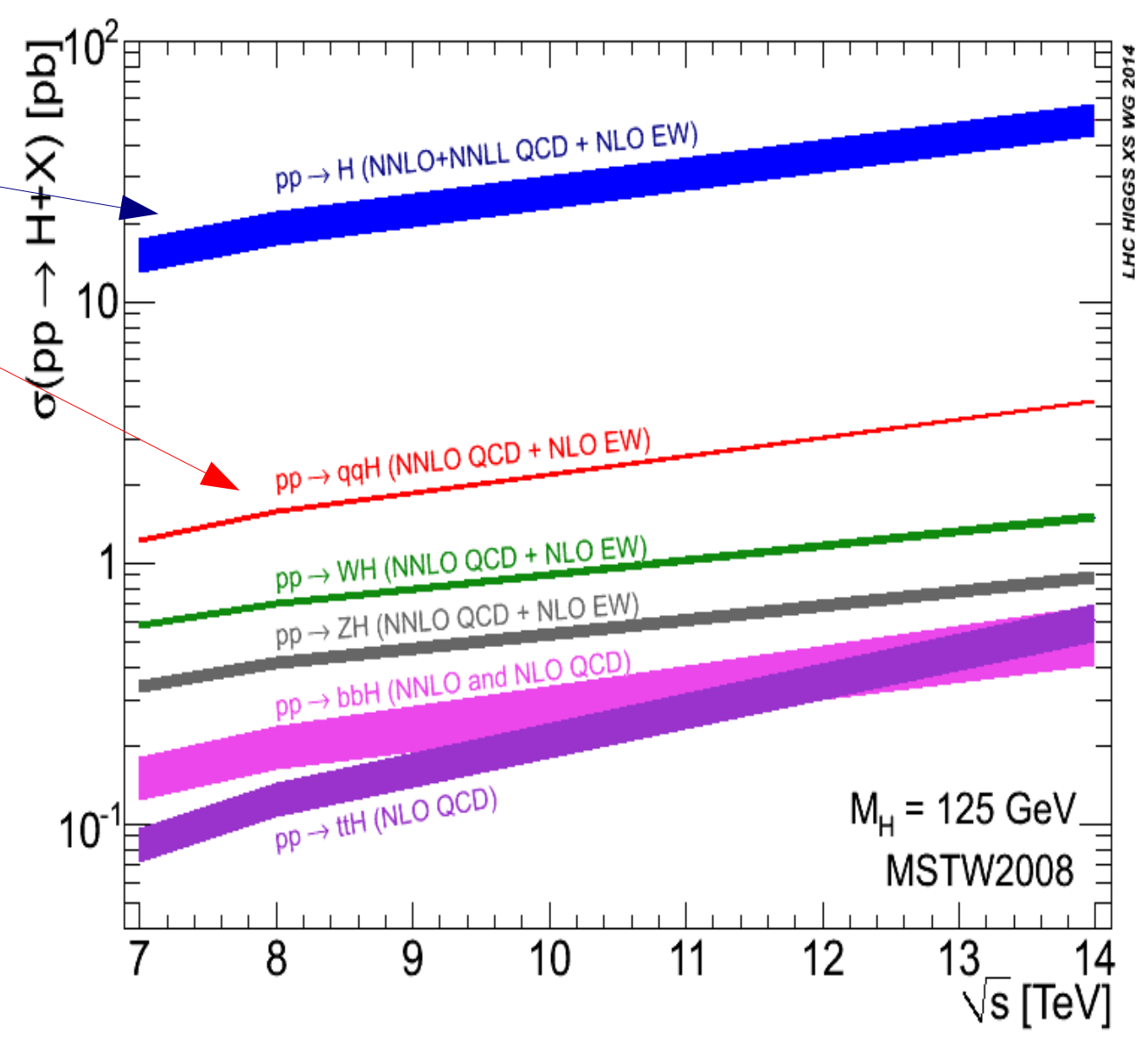




\section{Higgs decays}

Best mass resolution and good S/B

- $\mathrm{H} \rightarrow \mathrm{VY}$

- $\mathrm{H} \rightarrow \mathrm{ZZ}^{*} \rightarrow 4$ leptons

$$
M_{H}=125 \mathrm{GeV}
$$

\begin{tabular}{lc}
\hline \hline Decay channel & Branching ratio [\%] \\
\hline$H \rightarrow b b$ & $57.5 \pm 1.9$ \\
$H \rightarrow W W$ & $21.6 \pm 0.9$ \\
$H \rightarrow g g$ & $8.56 \pm 0.86$ \\
$H \rightarrow \tau \tau$ & $6.30 \pm 0.36$ \\
$H \rightarrow c c$ & $2.90 \pm 0.35$ \\
$H \rightarrow Z Z$ & $2.67 \pm 0.11$ \\
$H \rightarrow \gamma \gamma$ & $0.228 \pm 0.011$ \\
$H \rightarrow Z \gamma$ & $0.155 \pm 0.014$ \\
$H \rightarrow \mu \mu$ & $0.022 \pm 0.001$ \\
\hline \hline
\end{tabular}

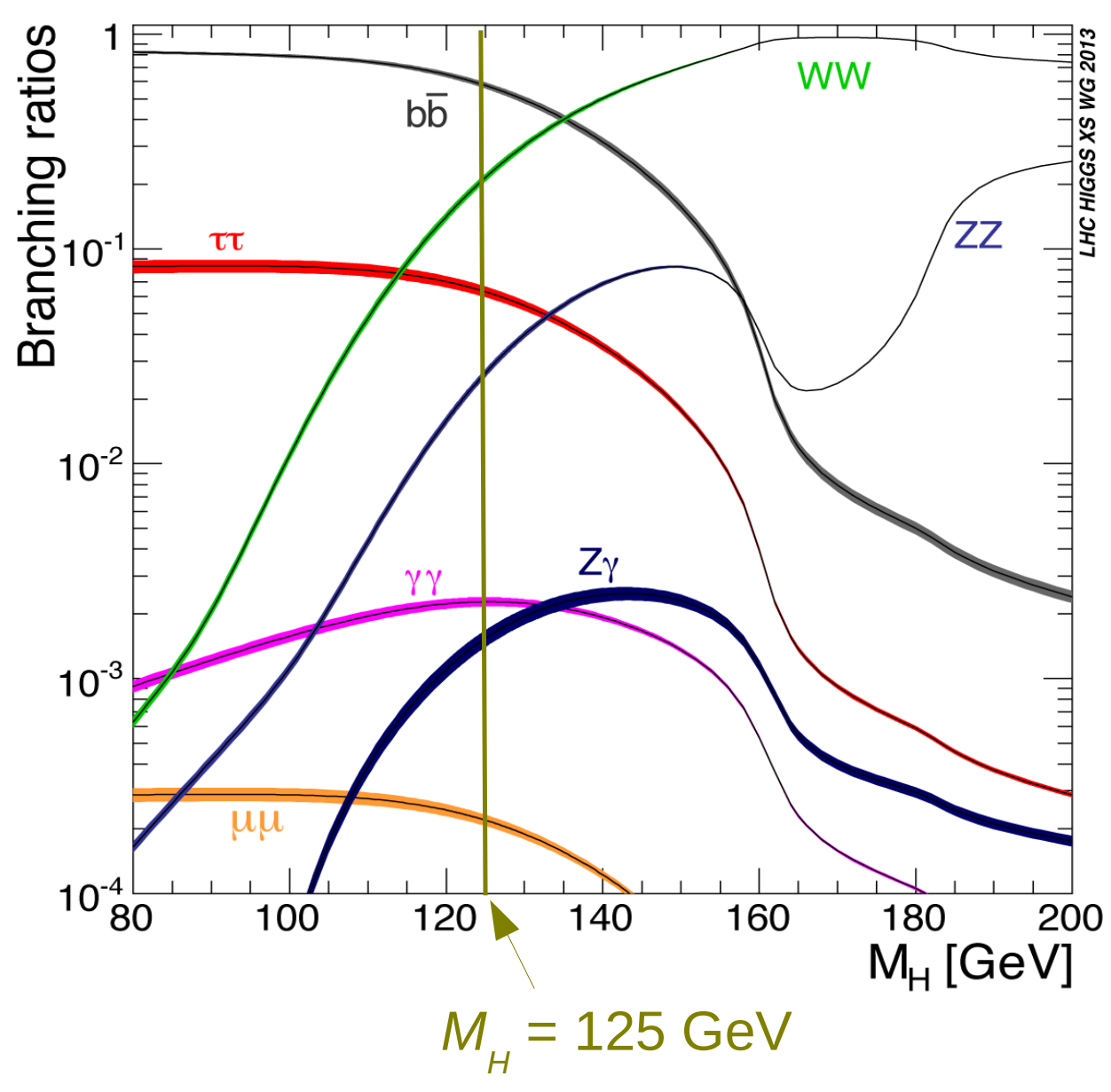




\section{Higgs boson at LHC}

New resonance with mass around

Phys. Lett. B 716(2012)

$125 \mathrm{GeV}$ announced by ATLAS \&

CMS experiments in July 2012
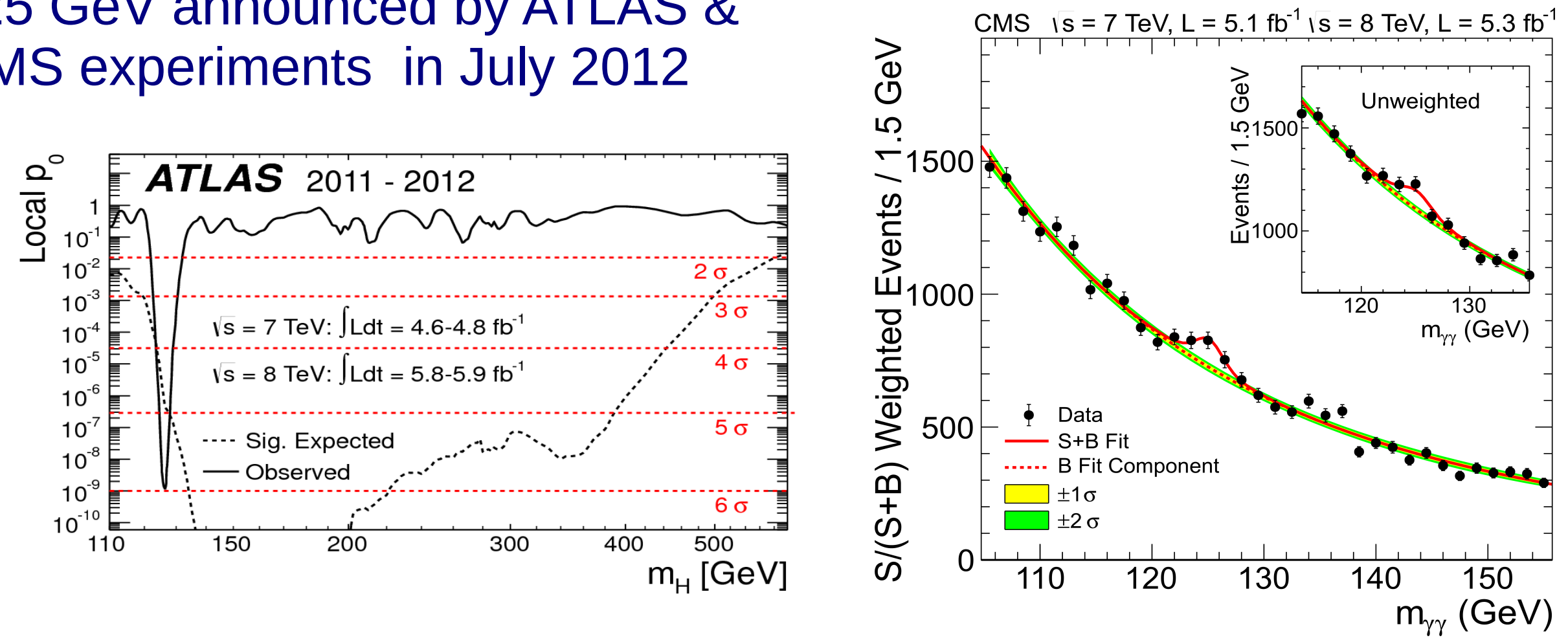

Measurements of its properties show good agreement with SM Higgs boson

Nobel prize for Physics 2013 to F. Englert, P. Higgs 


\section{Mass of the Higgs boson (I.)}

Phys. Rev. Lett. 114 (2015)

- Combination of ATLAS \& CMS measurements

- Channels with best mass resolution used $(\mathrm{H} \rightarrow \mathrm{yy}, \mathrm{H} \rightarrow$ 4leptons)

- Simultaneous fit to the reconstructed invariant mass peaks in the two channels and for the two experiments

- Mass measurement as independent as possible on the SM assumptions

- Maximization of profile likelihood ratios

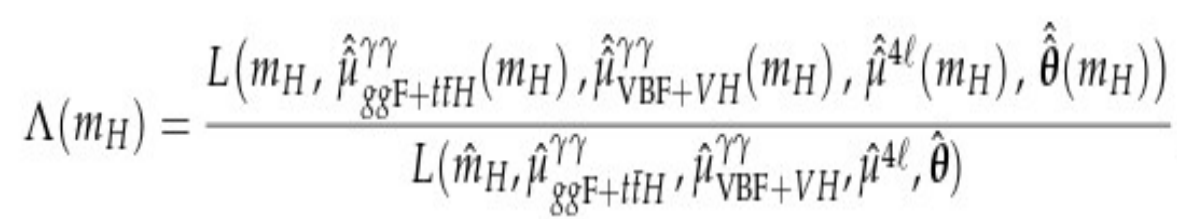

$\mu_{j}^{i}$ (signal strengths): ratio of experimental and SM expected signal yields

- two factors for $\mathrm{H} \rightarrow \mathrm{yy}$ (depending on the production mode) and one for $\mathrm{H} \rightarrow$ 4leptons

$\theta$ (nuisance parameters): systematic uncertainties 


\section{Mass of the Higgs boson (II.)}

Phys. Rev. Lett. 114 (2015)

Result:

$m_{H}=125.09 \pm 0.21$ (stat) \pm 0.11 (scale) \pm 0.02 (other) \pm 0.01 (theory) $\mathrm{GeV}$

- Total uncertainty dominated by statistical error

- Systematic uncertainty dominated by energy/momentum scale and resolution

Measurements are consistent with each other

- Between the different decay channels and between the two experiments

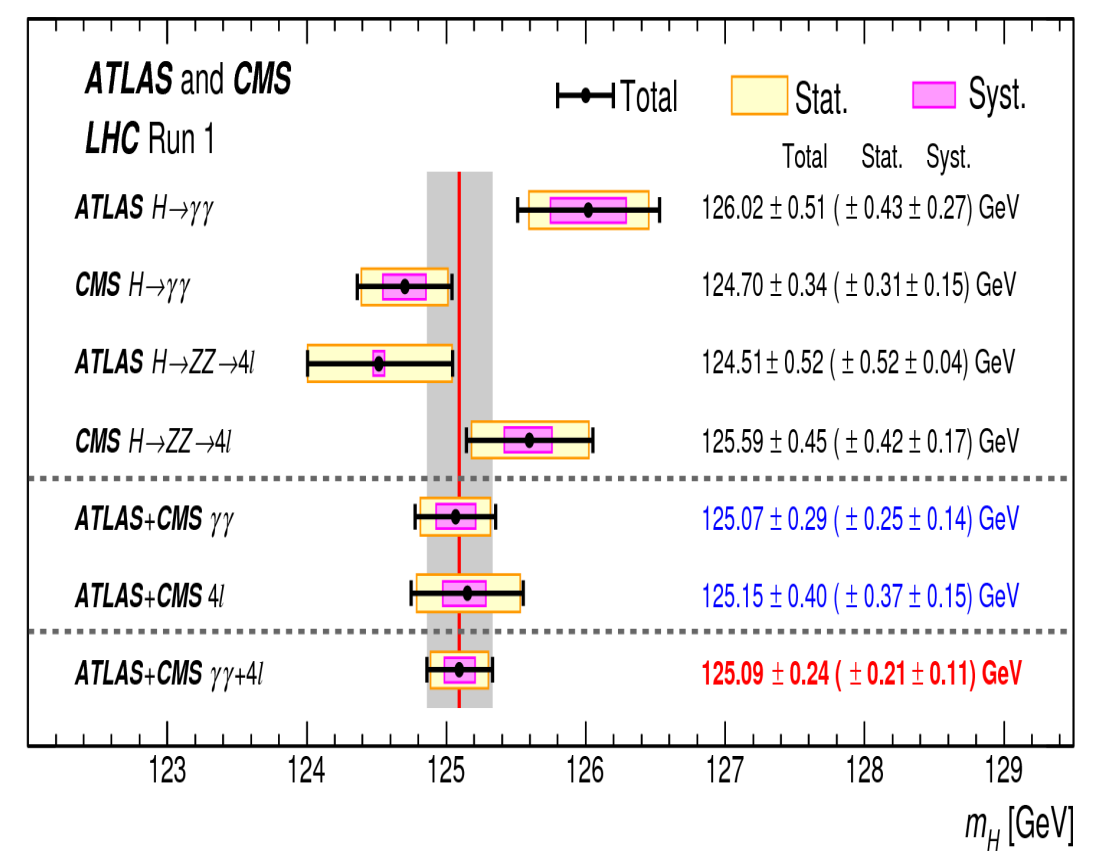




\section{Production and decay strength (I.)}

- Combination of ATLAS \& CMS measurements

- Production and decay signal strength $\mu_{i}=\frac{\sigma_{i}}{\left(\sigma_{i}\right)_{S M}}, \mu^{f}=\frac{B R^{f}}{\left(B R^{f}\right)_{S M}}$

- $\mu_{\mathrm{i}} \times \mu^{\mathrm{f}}$ measured experimentally

$$
\mu_{i}=\frac{\sigma_{i}}{\left(\sigma_{i}\right)_{S M}}, \mu^{f}=\frac{B R^{f}}{\left(B R^{f}\right)_{S M}}
$$

- Five production processes (ggH, VBF, WH, ZH, ttH)

- Six decay channels $(\mathrm{H} \rightarrow \mathrm{y}$,

\begin{tabular}{|c|c|c|c|c|c|c|}
\hline \multirow[t]{2}{*}{ Channel } & \multicolumn{2}{|c|}{$\begin{array}{l}\text { References for } \\
\text { individual publications }\end{array}$} & \multicolumn{4}{|c|}{$\begin{array}{l}\text { Signal strength }[\mu] \quad \text { Signal significance }[\sigma] \\
\text { from results in this paper (Section 5.2) }\end{array}$} \\
\hline & ATLAS & CMS & ATLAS & CMS & ATLAS & CMS \\
\hline$H \rightarrow \gamma \gamma$ & [51] & {$[52]$} & $\begin{array}{c}1.15_{-0.25}^{+0.27} \\
\left(\begin{array}{c}+0.26 \\
-0.24\end{array}\right)\end{array}$ & $\begin{array}{c}1.12_{-0.23}^{+0.25} \\
\left(\begin{array}{l}+0.24 \\
(-0.22\end{array}\right)\end{array}$ & $\begin{array}{c}5.0 \\
(4.6) \\
\end{array}$ & $\begin{array}{c}5.6 \\
(5.1) \\
\end{array}$ \\
\hline$H \rightarrow Z Z \rightarrow 4 \ell$ & [53] & [54] & $\begin{array}{c}1.51_{-0.34}^{+0.39} \\
\left(\begin{array}{c}+0.33 \\
-0.27\end{array}\right)\end{array}$ & $\begin{array}{l}1.05_{-0.27}^{+0.32} \\
\left(\begin{array}{c}+0.31 \\
-0.26\end{array}\right)\end{array}$ & $\begin{array}{c}6.6 \\
(5.5)\end{array}$ & $\begin{array}{c}7.0 \\
(6.8)\end{array}$ \\
\hline$H \rightarrow W W$ & {$[55,56]$} & [57] & $\begin{array}{c}1.23_{-0.21}^{+0.23} \\
\left(\begin{array}{c}+0.21 \\
-0.20\end{array}\right)\end{array}$ & $\begin{array}{l}0.91_{-0.21}^{+0.24} \\
\left(\begin{array}{c}+0.23 \\
-0.20\end{array}\right)\end{array}$ & 6.8 & 4.8 \\
\hline$H \rightarrow \tau \tau$ & {$[58]$} & [59] & $\begin{array}{c}1.41_{-0.35}^{+0.40} \\
\left(\begin{array}{l}+0.37 \\
-0.33\end{array}\right)\end{array}$ & $\begin{array}{c}0.89_{-0.28}^{+0.31} \\
\left(\begin{array}{c}+0.31 \\
-0.29\end{array}\right)\end{array}$ & $\begin{array}{c}4.4 \\
(3.3)\end{array}$ & $\begin{array}{c}3.4 \\
(3.7)\end{array}$ \\
\hline$H \rightarrow b b$ & {$[38]$} & [39] & $\begin{array}{c}0.62_{-0.36}^{+0.37} \\
\left(\begin{array}{c}+0.39 \\
-0.37\end{array}\right) \\
\end{array}$ & $\begin{array}{c}0.81_{-0.42}^{+0.45} \\
\left(\begin{array}{c}+0.45 \\
-0.43\end{array}\right)\end{array}$ & $\frac{1.7}{(2.7)}$ & $\frac{2.0}{(2.5)}$ \\
\hline$H \rightarrow \mu \mu$ & [60] & [61] & $\begin{array}{c}-0.7 \pm 3.6 \\
( \pm 3.6)\end{array}$ & $\begin{array}{c}0.8 \pm 3.5 \\
( \pm 3.5)\end{array}$ & & \\
\hline$t t H$ production & {$[28,62,63]$} & [65] & $\begin{array}{l}1.9_{-0.7}^{+0.8} \\
\left(\begin{array}{c}+0.72 \\
-0.66\end{array}\right)\end{array}$ & $\begin{array}{c}2.9_{-0.9}^{+1.0} \\
\left(\begin{array}{c}+0.88 \\
-0.80\end{array}\right)\end{array}$ & $\begin{array}{c}2.7 \\
(1.6)\end{array}$ & $\begin{array}{c}3.6 \\
(1.3)\end{array}$ \\
\hline
\end{tabular}




\section{Production and decay strength (II.)}

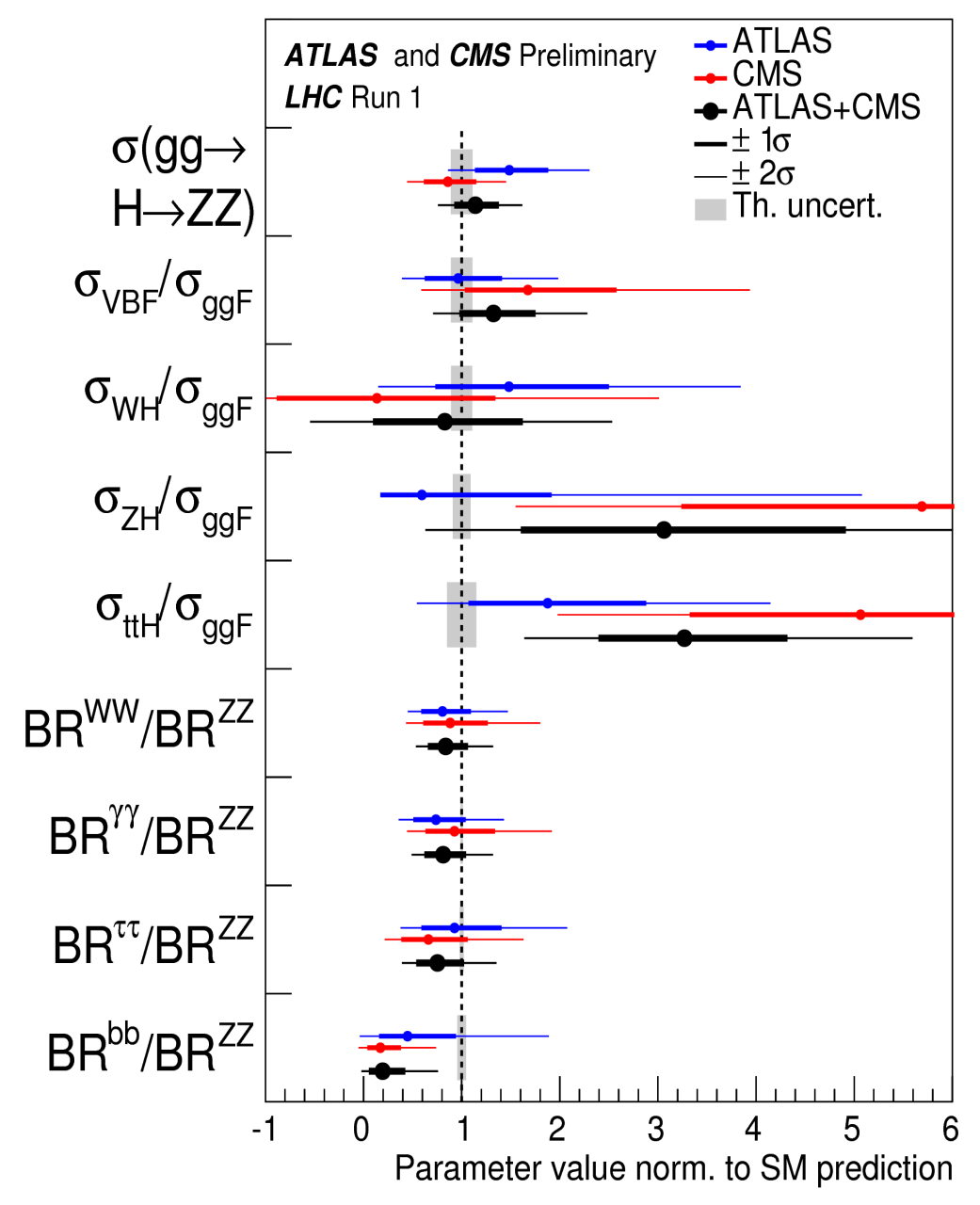

ATLAS-CONF-2015-044 CMS-PAS-HIG-15-002

- Combined signal yield relative to SM expectation $1.09 \pm 0.11$

- Observed significance of the VBF production mode at the level of $5.4 \sigma$ (4.7 expected)

- $\mathrm{H} \rightarrow \tau \tau$ channel at the level of $5.5 \sigma$ (5.0 expected)

\section{Data consistent with SM expectations}

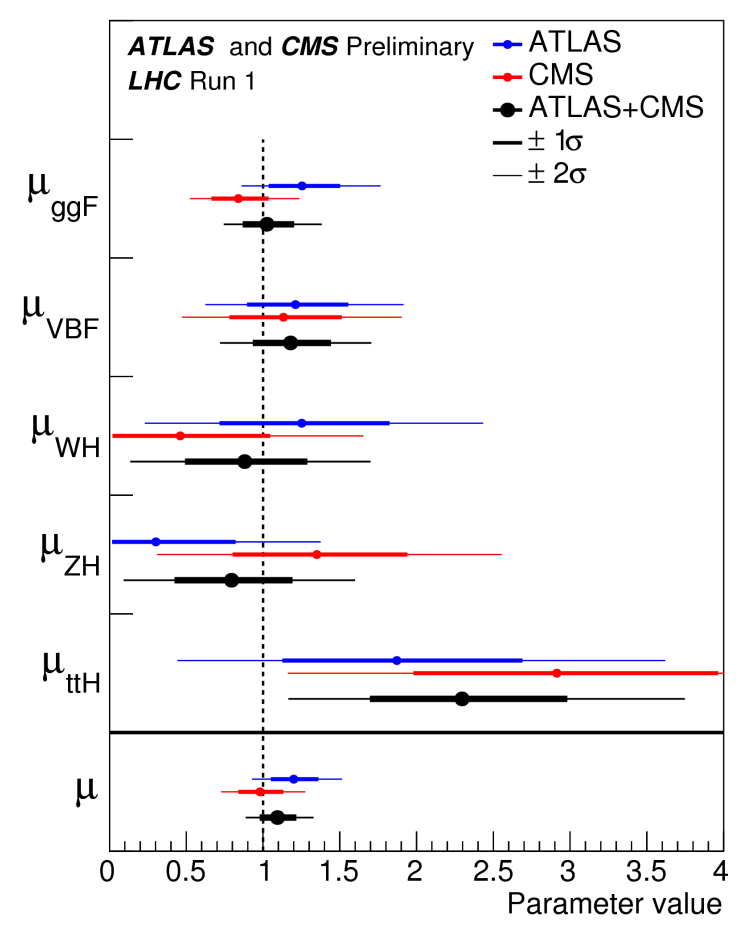




\section{Constraints on Higgs boson couplings}

- Testing of the couplings by allowing more generic models (BSM)

- Example: Parameterizations allowing contributions from BSM particles in loops and in decays

- Fitting of 7 coupling modifiers $k_{X}+B R_{B S M}$

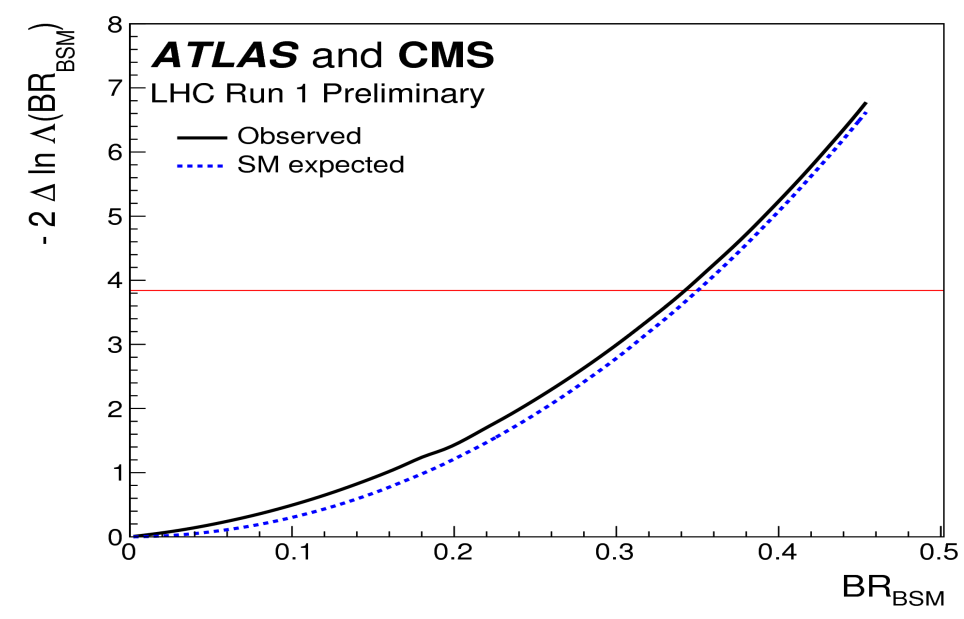

Data consistent with SM expectations

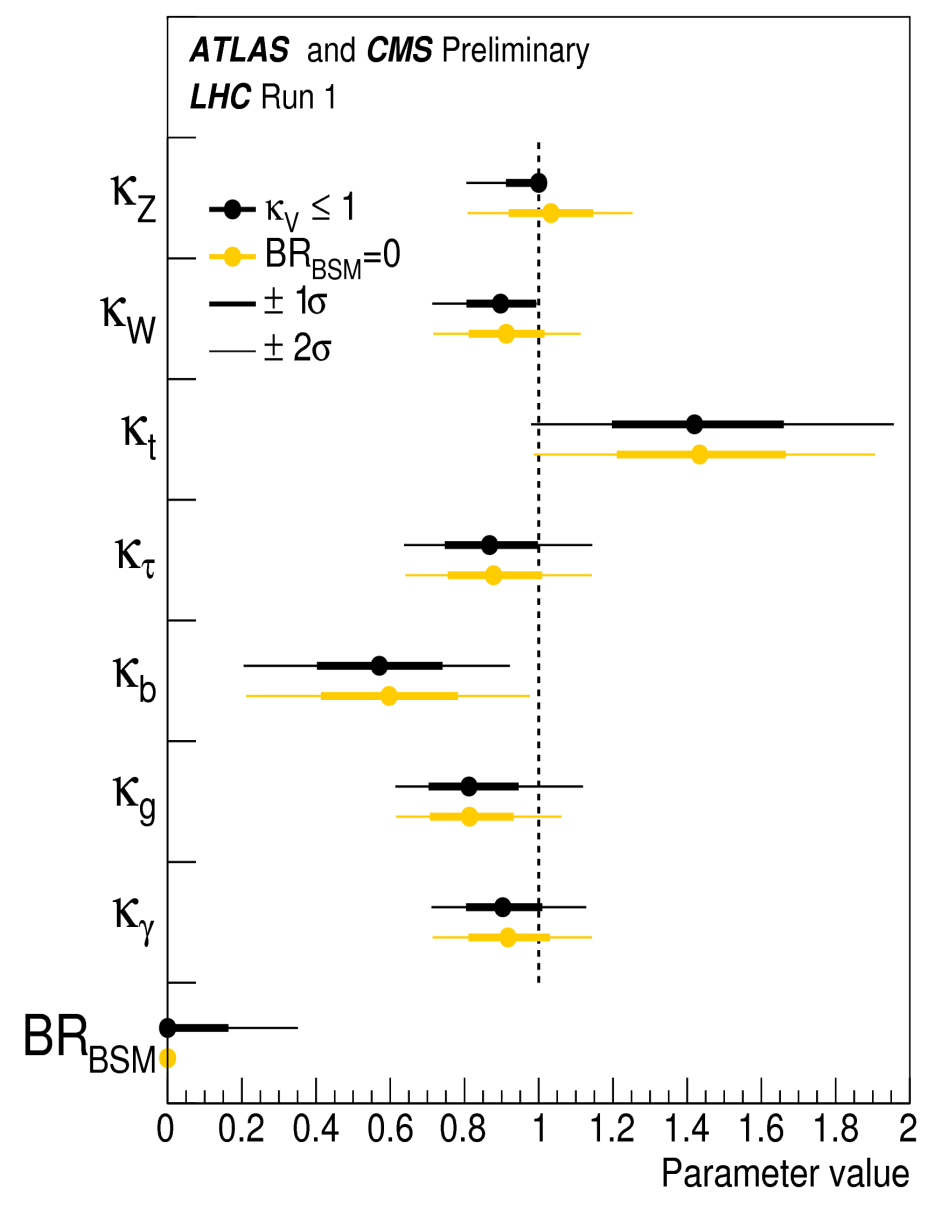




\section{Spin \& parity measurements (I.)}

Phys. Rev. D 92 (2015)

- CMS measurements using the $\mathrm{H} \rightarrow$ 4leptons, $\mathrm{H} \rightarrow \mathrm{WW} \rightarrow \ell v \ell v$, and $\mathrm{H} \rightarrow \mathrm{Vy}$ decay modes

- Different variables sensitive to the spin and parity of the Higgs boson built

- Examples from $\mathrm{H} \rightarrow$ 4leptons channel
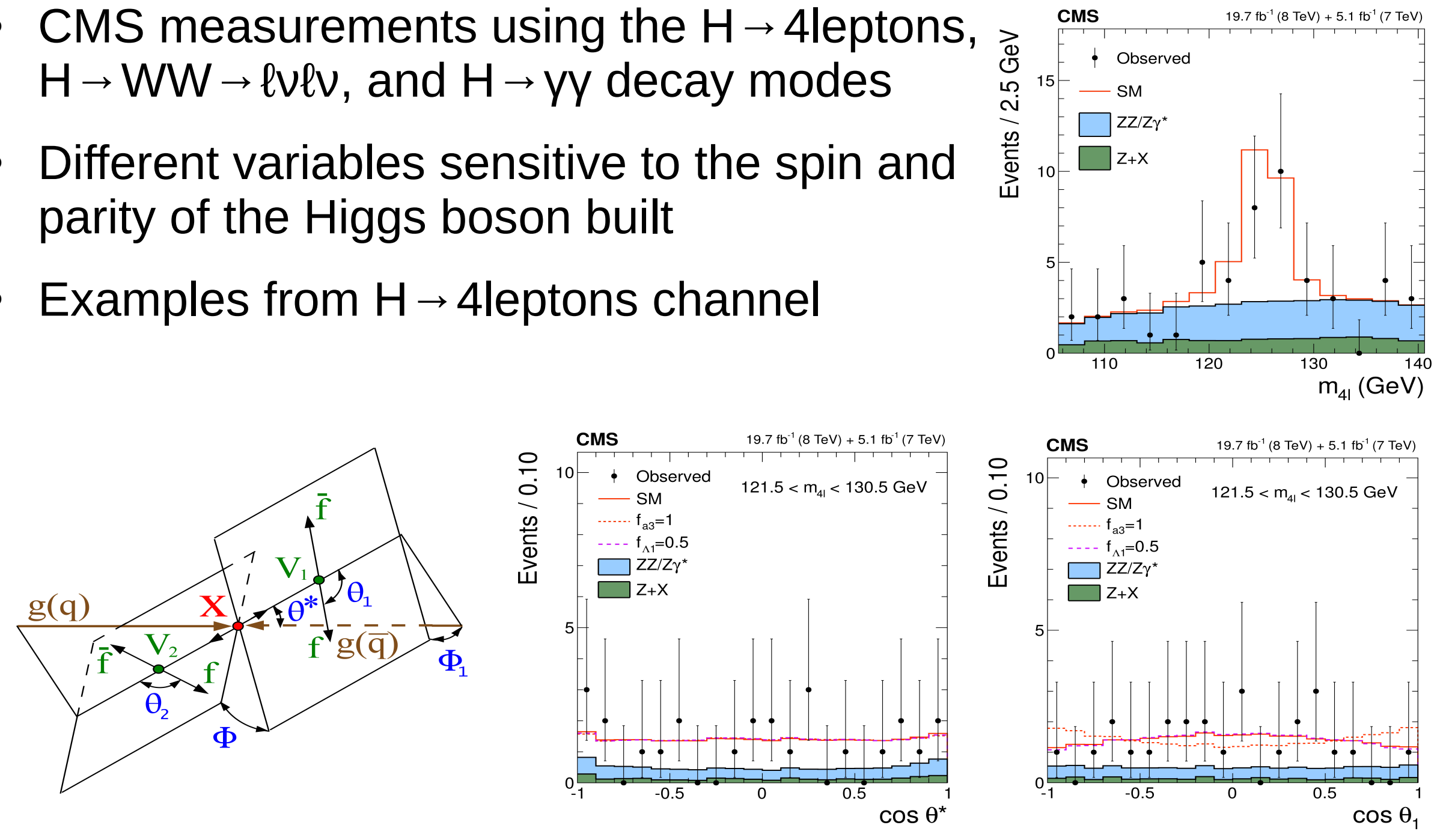


\section{Spin \& parity measurements (II.)}

- Wide range of spin-two models excluded at 99\% confidence level or higher

- Mixed-parity spin-one state is excluded at a confidence level of $99.999 \%$

- All observations consistent with the expectations for a scalar SM-like Higgs boson $\left(\mathrm{J}^{\mathrm{PC}}=\mathrm{0}^{++}\right)$

\section{Phys. Rev. D 92 (2015)}

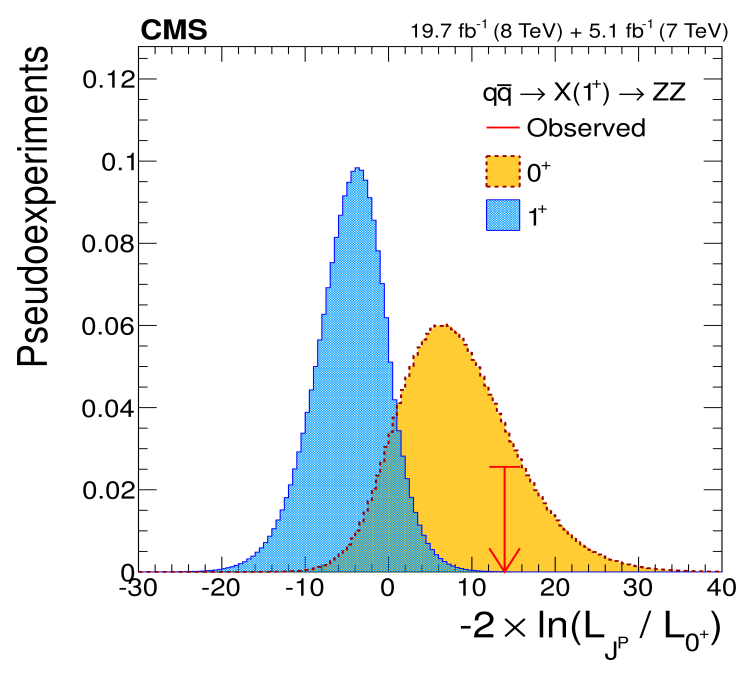

- Similar conclusions in ATLAS

Eur. Phys. J. C75 (2015)

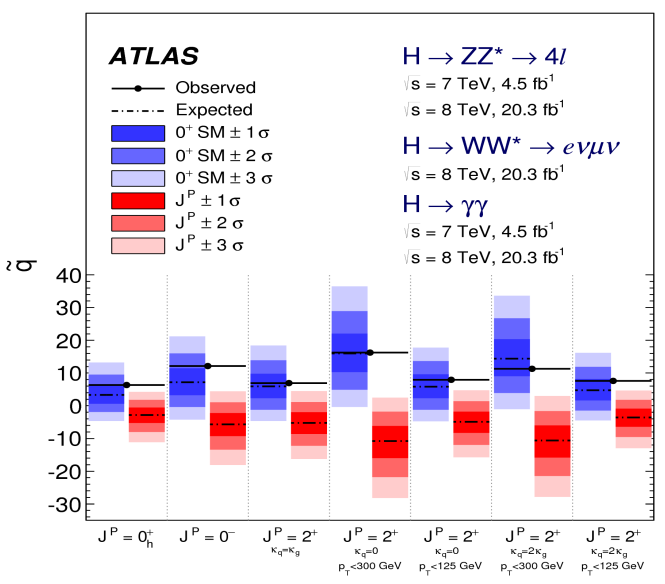




\section{Summary of Run1 measurements}

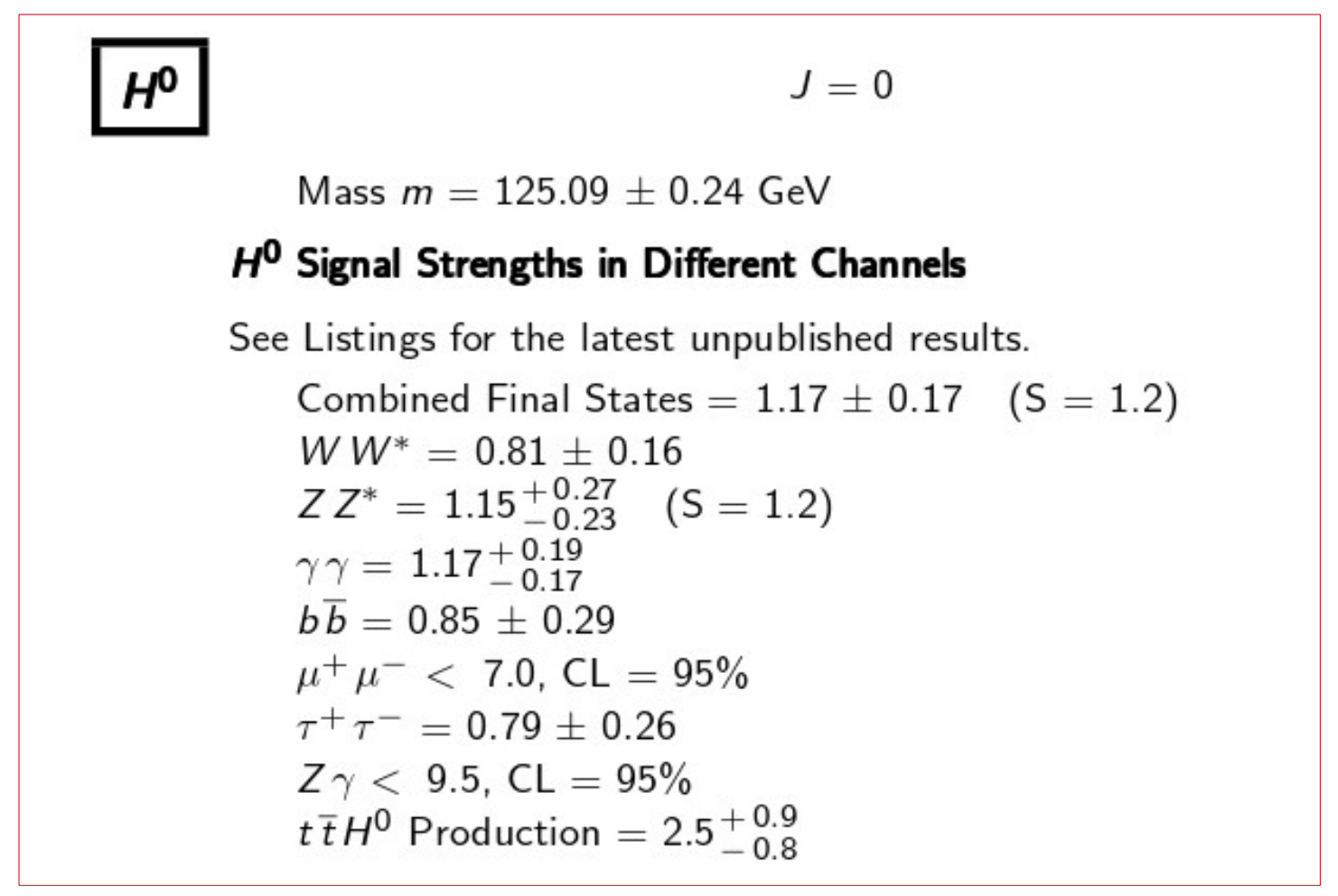

All results in agreement with the SM expectations 


\section{Analysis with current Run2 dataset}

- Limited statistics compared to Run1 so far

- CMS

- Preparation stage for the analysis with more data

- Trigger, ID, selection efficiencies

- Control regions checks

- All Higgs boson analysis blinded at the moment

- ATLAS

- Cross-section measurements with discovery channels $(H \rightarrow y y$, $\mathrm{H} \rightarrow$ 4leptons)

- Preparation stage for the rest of the analysis 


\section{$\mathrm{H} \rightarrow \mathrm{yy}$ : Performance studies at CMS}

- Search for a pair of well identified photons
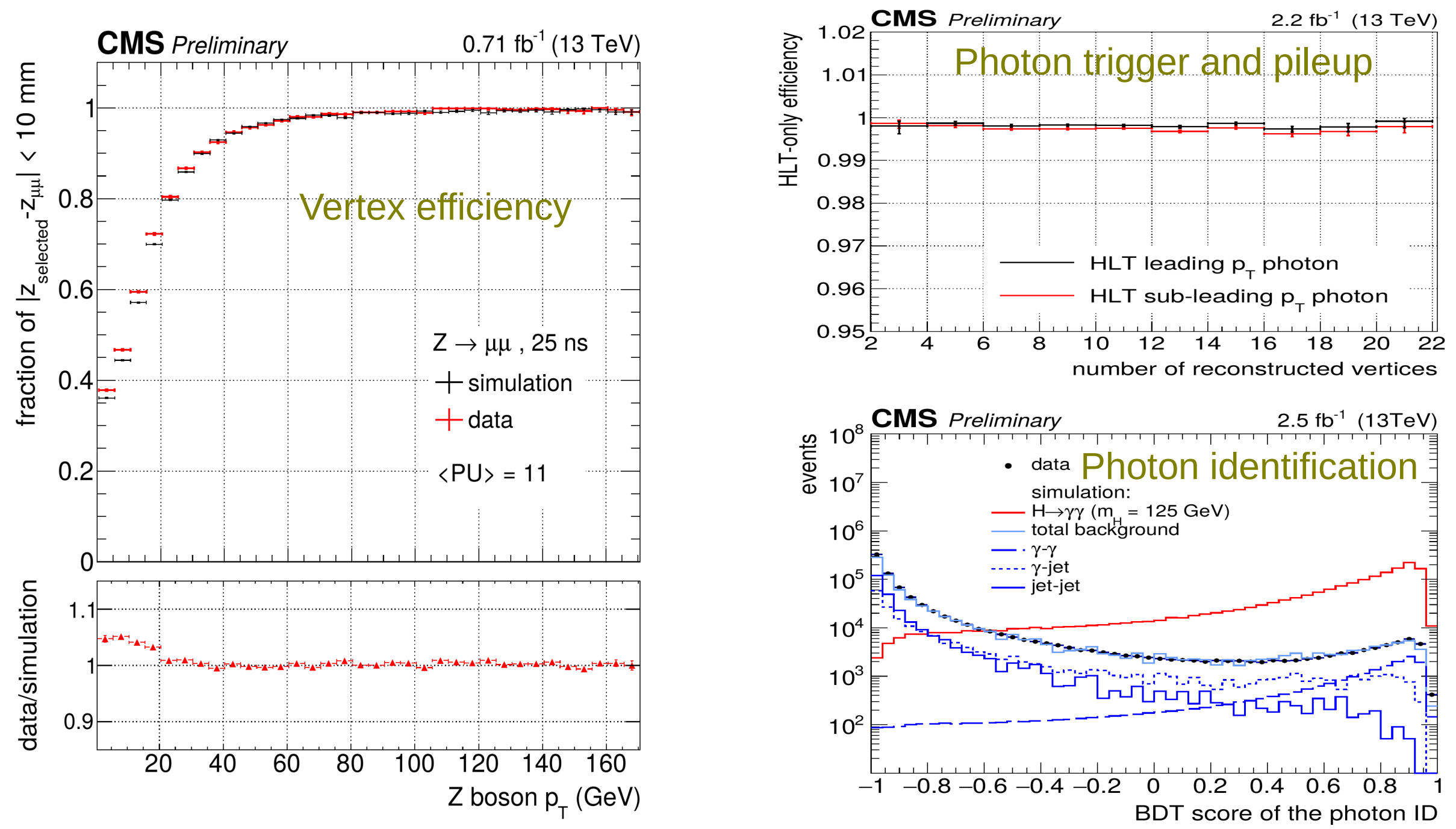


\section{Cross-section measurement}

$$
\sigma=\frac{N_{S}}{A C L_{i}} \quad \begin{aligned}
& \sigma-\text { cross-section } \\
& N_{s} \text { - extracted signal yield } \\
& \begin{array}{l}
A-\text { acceptance factor } \\
L_{i} \text { - integrated luminosity }
\end{array}
\end{aligned}
$$

- Correction factor $(C)$

- Accounts for detector resolution and efficiency

- Acceptance factor $(A)$

- Efficiency of selection cuts at truth level 


\section{$\mathrm{H} \rightarrow \mathrm{VY}$ analysis at ATLAS (I.)}

ATLAS-CONF-2015-060

- Event selection

- Two tight identified \& isolated photons

- Relative transverse energy cut $\left(E_{T} / m_{y y}>0.35 / 0.25\right)$
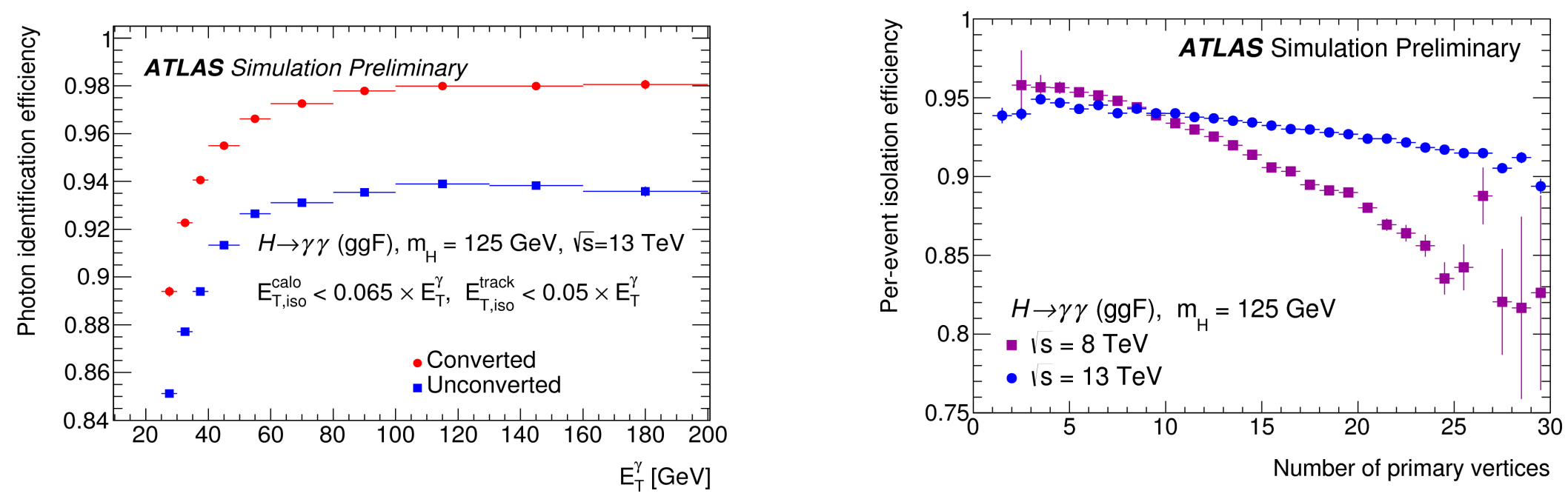

- Signal selection efficiency between 31 and 37\% depending on the production mode 


\section{$\mathrm{H} \rightarrow \mathrm{yY}$ analysis at ATLAS (II.)}

- Main backgrounds

- yy continuum

- $\mathrm{y}+$ jet, jet+jet production

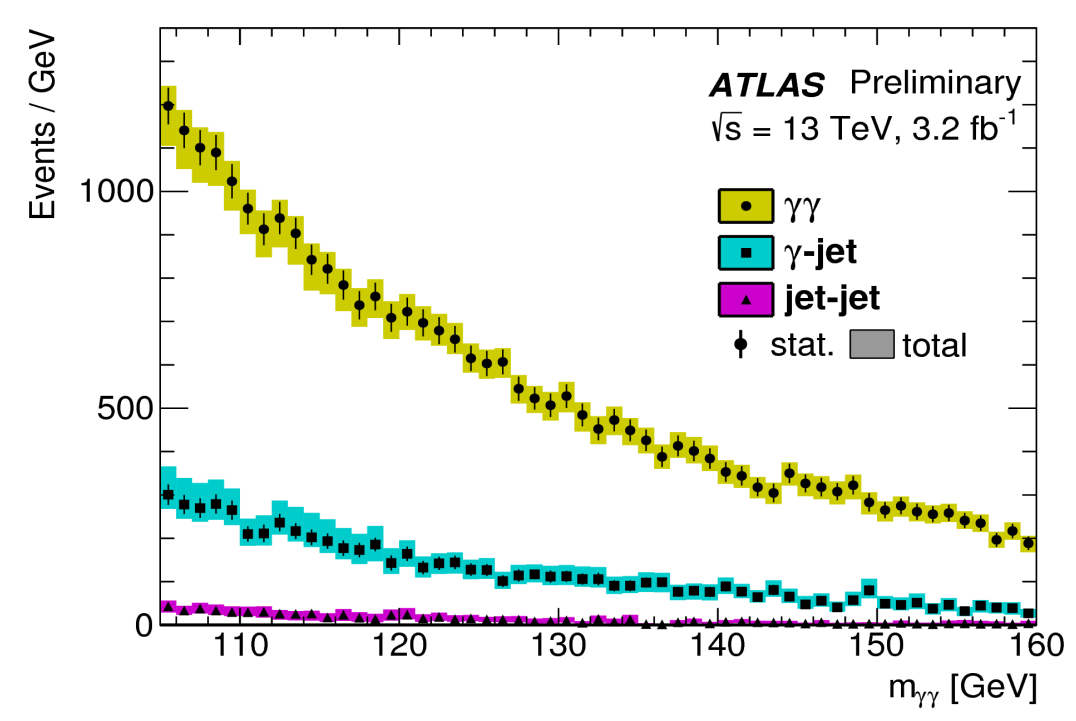

- Diphoton invariant mass spectrum fitted with signal + background model for the fixed Higgs boson mass $125.09 \mathrm{GeV}$

$$
\begin{aligned}
& N_{\text {exp }}=143 \pm 71 \text { (stat.) } \pm{ }_{6}^{39} \text { (syst.) } \\
& N_{s}=113 \pm 74 \text { (stat.) } \pm{ }_{25}^{43} \text { (syst.) }
\end{aligned}
$$

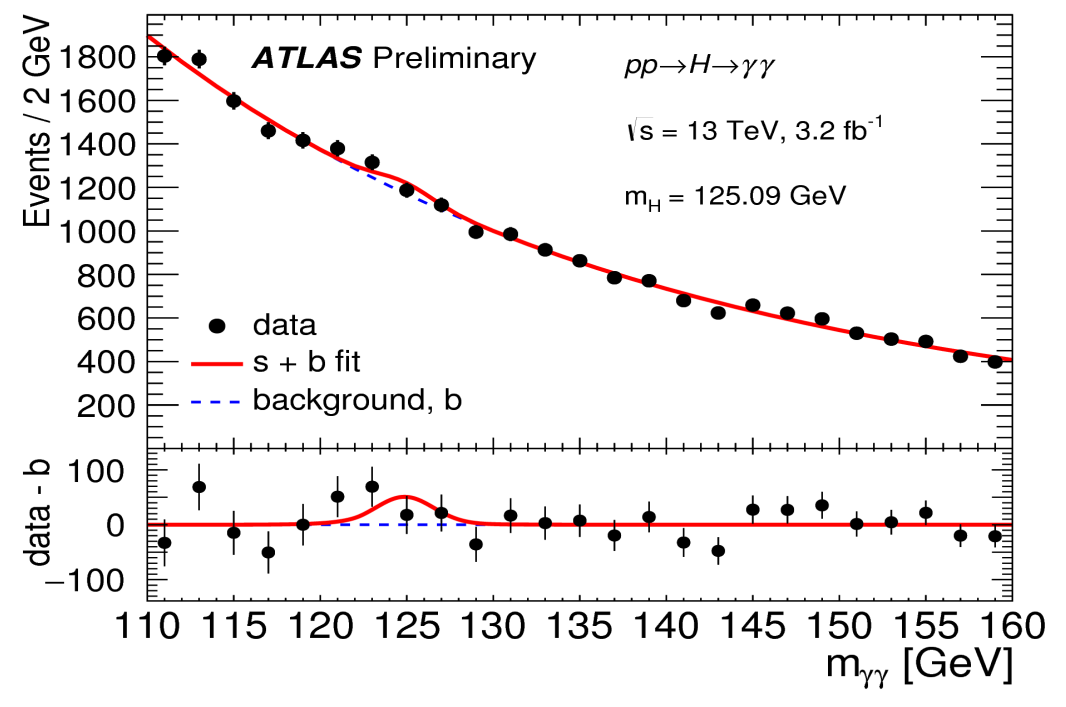




\section{$\mathrm{H} \rightarrow \mathrm{yy}$ cross-section (I.)}

- Correction factor

- Defined as ratio of the expected number of events passing the selection requirement with the number of generated particle-level events in the fiducial volume

- $C=0.68$

\begin{tabular}{l|l}
\hline \multicolumn{2}{c}{ Event Selection } \\
\hline Two highest- $p_{\mathrm{T}}$ photons: & $\left|\eta^{\gamma}\right|<2.37$ \\
Relative- $p_{\mathrm{T}}:$ & $E_{\mathrm{T}, 1}^{\gamma} / m_{\gamma \gamma} \geq 0.35, E_{\mathrm{T}, 2}^{\gamma} / m_{\gamma \gamma} \geq 0.25$ \\
Mass window: & $105 \mathrm{GeV} \leq m_{\gamma \gamma}<160 \mathrm{GeV}$ \\
Photon isolation: & $E_{\mathrm{T}, \text { iso }}<0.1 \times E_{\mathrm{T}}^{\gamma}+1 \mathrm{GeV}$ \\
\hline
\end{tabular}

\begin{tabular}{lc}
\hline Component & Uncertainty [\%] \\
\hline Photon energy scale & $<0.1$ \\
Photon energy resolution & $<0.1$ \\
Photon identification efficiency & \pm 2.6 \\
Photon isolation efficiency & \pm 4.0 \\
Trigger efficiency & \pm 0.4 \\
Vertex selection & $<0.1$ \\
\hline Theoretical modeling uncertainty & \pm 0.8 \\
\hline Total & \pm 4.8 \\
\hline
\end{tabular}




\section{$\mathrm{H} \rightarrow \mathrm{yy}$ cross-section (II.)}

- Acceptance factor

- Fraction of $\mathrm{H} \rightarrow \mathrm{yy}$ decays that pass the fiducial selection

\begin{tabular}{cc}
\hline$\sqrt{s}$ & $\mathcal{A}$ \\
\hline $7 \mathrm{TeV}$ & $0.620 \pm 0.007$ \\
$8 \mathrm{TeV}$ & $0.611 \pm 0.012$ \\
$13 \mathrm{TeV}$ & $0.570 \pm 0.006$ \\
\hline
\end{tabular}

- Cross-section results

\begin{tabular}{ccc}
\hline$\sqrt{s}$ & Measured total cross section $[\mathrm{pb}]$ & LHC-XS prediction $[\mathrm{pb}]$ \\
\hline $7 \mathrm{TeV}$ & $35 \pm 12$ (stat.) \pm 4 (syst.) \pm 1 (lumi.) & $17.5 \pm 1.6$ \\
$8 \mathrm{TeV}$ & $30.5 \pm 7.1$ (stat.) ${ }_{-2.5}^{+2.6}$ (syst.) \pm 0.9 (lumi.) & $22.3 \pm 2.0$ \\
$13 \mathrm{TeV}$ & $40 \pm 26$ (stat.) ${ }_{-10}^{+16}$ (syst.) \pm 2 (lumi.) & $50.9_{-4.4}^{+4.5}$ \\
\hline
\end{tabular}




\section{$\mathrm{H} \rightarrow$ 4leptons at CMS}

- Measurement of the fiducial cross section of the dominant background (ZZ* continuum)

- Blinded analysis at the moment

- Mass spectrum matches well the expectations in the side band regions

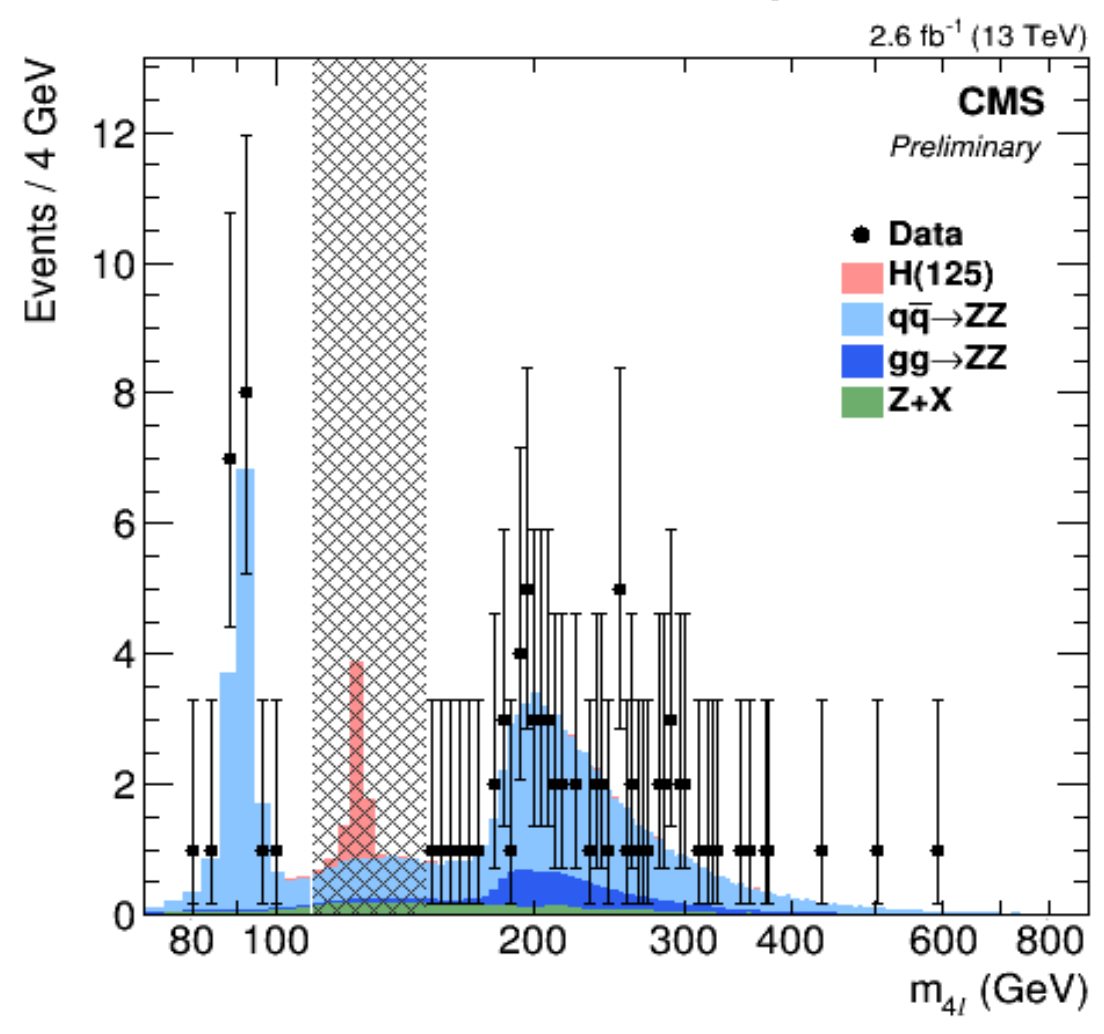




\section{$\mathrm{H} \rightarrow$ 4leptons analysis at ATLAS (I.)}

ATLAS-CONF-2015-059

Event with 4 identified \& isolated leptons and 2 jets

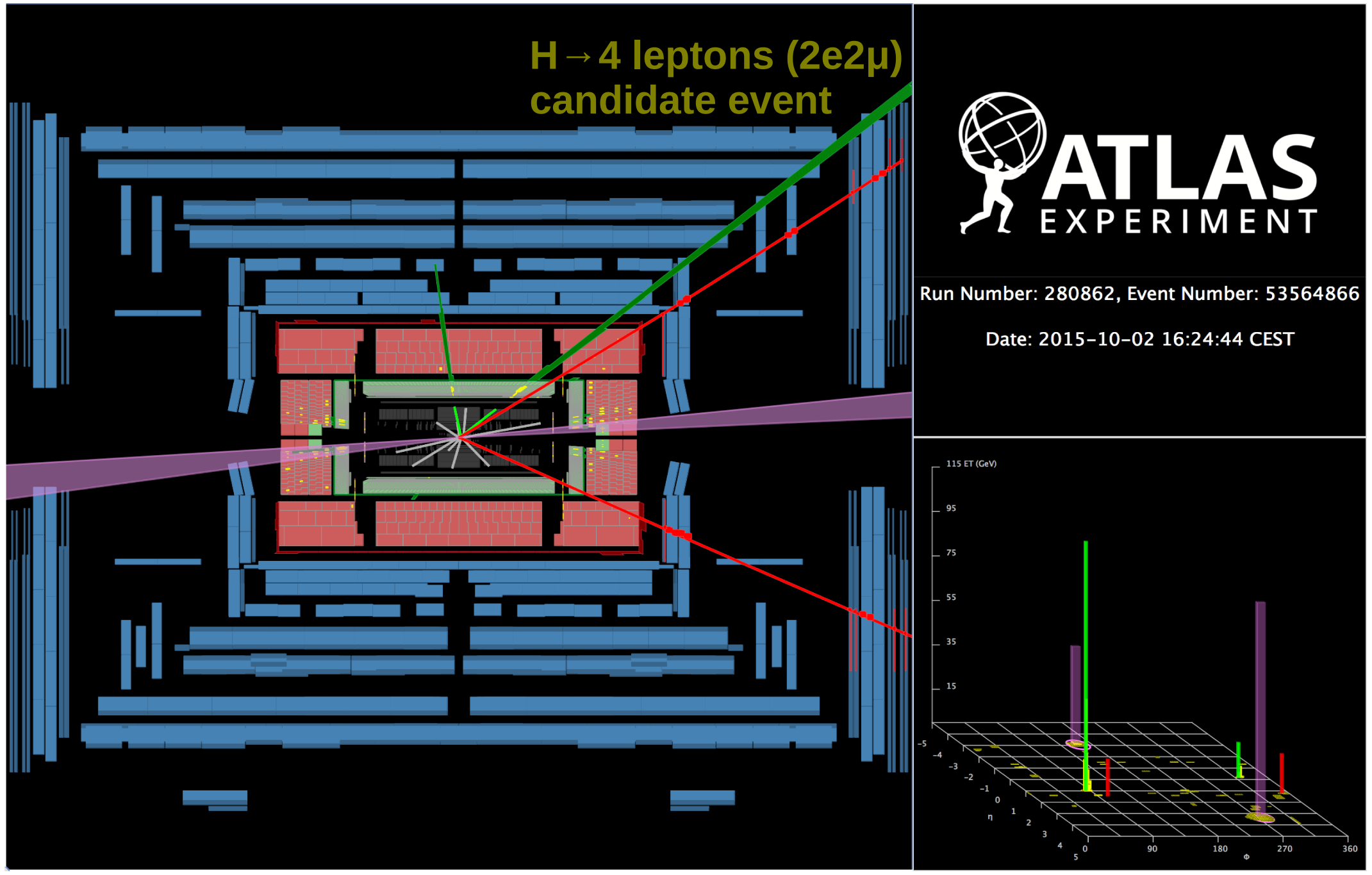




\section{$\mathrm{H} \rightarrow$ 4leptons analysis at ATLAS (II.)}

- Main backgrounds

- ZZ* non-resonant diboson production

- Z+jets, t-tbar
$80 \mathrm{GeV}<m_{4 \mid}<170 \mathrm{GeV}$

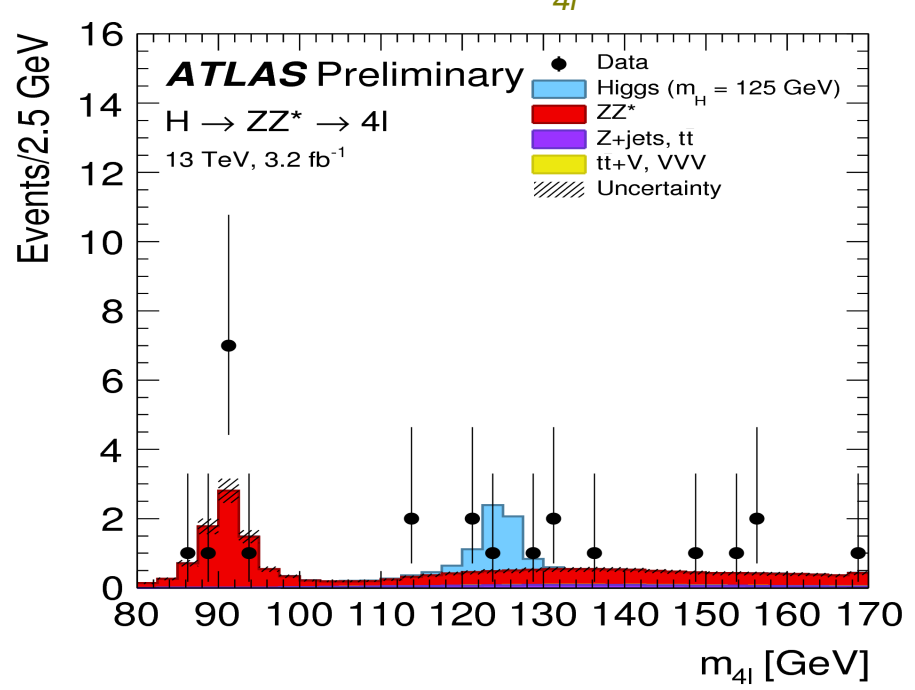

$160 \mathrm{GeV}<m_{41}<1000 \mathrm{GeV}$

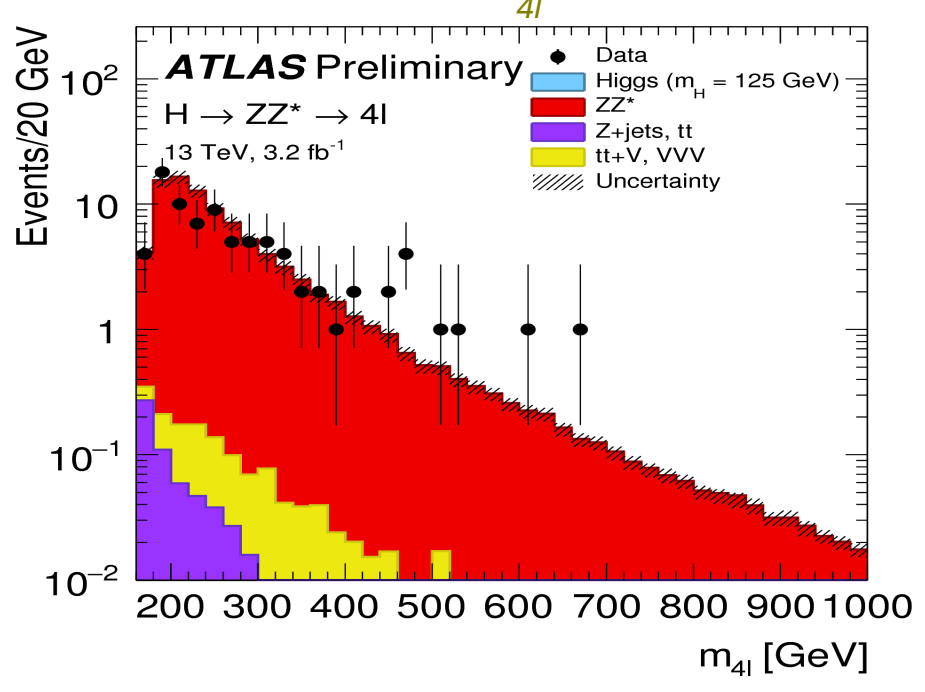

\begin{tabular}{cccccccc}
\hline \hline Final state & $\begin{array}{c}\text { Signal } \\
\text { full mass range }\end{array}$ & Signal & $Z Z^{*}$ & $\begin{array}{c}Z+\mathrm{jets}, t \bar{t} \\
t \bar{t} V, V V V, W Z\end{array}$ & $S / B$ & Expected & Observed \\
\hline $4 \mu$ & $1.79 \pm 0.21$ & $1.67 \pm 0.20$ & $0.64 \pm 0.06$ & $0.08 \pm 0.03$ & 2.3 & $2.39 \pm 0.21$ & 1 \\
$2 e 2 \mu$ & $1.19 \pm 0.14$ & $1.06 \pm 0.13$ & $0.44 \pm 0.04$ & $0.07 \pm 0.03$ & 2.1 & $1.57 \pm 0.14$ & 1 \\
$2 \mu 2 e$ & $1.07 \pm 0.16$ & $0.96 \pm 0.15$ & $0.34 \pm 0.05$ & $0.09 \pm 0.02$ & 2.2 & $1.40 \pm 0.16$ & 2 \\
$4 e$ & $1.01 \pm 0.15$ & $0.88 \pm 0.13$ & $0.32 \pm 0.05$ & $0.09 \pm 0.02$ & 2.1 & $1.30 \pm 014$ \\
\hline Total & $5.06 \pm 0.60$ & $4.57 \pm 0.54$ & $1.74 \pm 0.19$ & $0.34 \pm 0.06$ & 2.2 & $6.65 \pm 0.58$ \\
\hline \hline
\end{tabular}




\section{$\mathrm{H} \rightarrow$ 4leptons cross-section}

Acceptance \& correction factor

\begin{tabular}{lrccc}
\hline & \multicolumn{3}{c}{$\sqrt{s}[\mathrm{TeV}]$} \\
\cline { 2 - 4 } $\mathcal{A}[\%]$ & 7 & 8 & 13 \\
\cline { 2 - 4 } $\mathcal{C}[\%]$ & $46.67 \pm 0.23$ & $45.98 \pm 0.14$ & $42.74 \pm 0.24$ \\
$\mathcal{C}$ & $51.89 \pm 0.36$ & $55.32 \pm 0.24$ & $52.71 \pm 0.45$ \\
\hline
\end{tabular}

Cross-section results

\begin{tabular}{cccccc}
\hline \hline Data set $[\mathrm{TeV}]$ & $N_{\mathrm{s}}$ & $\sigma_{4 \ell}^{\text {fid }}[\mathrm{fb}]$ & $\sigma_{\text {theory }}^{\text {fid }}[\mathrm{fb}]$ & $\sigma^{\text {tot }}[\mathrm{pb}]$ & $\sigma_{\text {theory }}^{\text {tot }}[\mathrm{pb}]$ \\
\hline 7 & $4.5_{-2.2}^{+2.8}$ & $1.9_{-0.9}^{+1.2}$ & $1.03 \pm 0.11$ & $33_{-16}^{+21}$ & $17.5 \pm 1.6$ \\
8 & $24.0_{-5.3}^{+6.0}$ & $2.1 \pm 0.5$ & $1.29 \pm 0.13$ & $37_{-8}^{+9}$ & $22.3 \pm 2.0$ \\
13 & $1.0_{-1.5}^{+2.3}$ & $0.6_{-0.9}^{+1.3}$ & $2.74 \pm 0.28$ & $12_{-16}^{+25}$ & $50.9_{-4.4}^{+4.5}$ \\
\hline \hline
\end{tabular}




\section{Cross-section combination}

ATLAS-CONF-2015-069

- Combination of $\mathrm{H} \rightarrow \mathrm{yy}$ and $\mathrm{H} \rightarrow$ 4leptons cross-section measurements

- Comparison of 7, 8 and $13 \mathrm{TeV}$ results

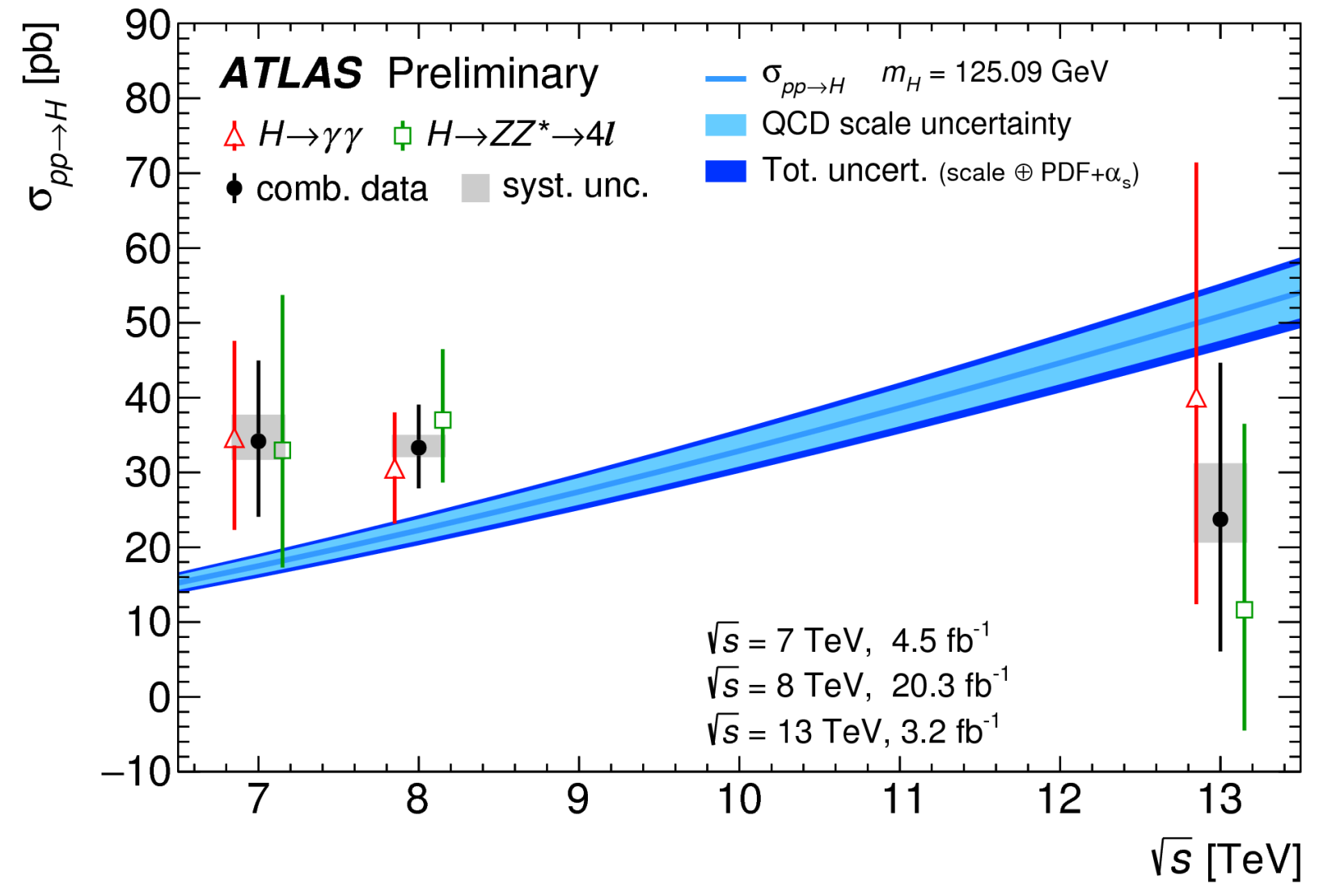




\section{Conclusions}

- Run1 measurements consistent with the SM expectations

- First combined measurements by ATLAS and CMS for mass and couplings

- First Higgs boson measurements in Run2 performed with the ATLAS detector

- Channels $\mathrm{H} \rightarrow \mathrm{yy}$ and $\mathrm{H} \rightarrow$ 4leptons

- Preparation of the analysis for larger dataset in ATLAS and CMS experiments

- Much more to come with more data

- Different channels

- CP, production \& decay rates, differencial cross-section

- Rare decays 